\title{
13. CALCAREOUS NANNOFOSSIL BIOSTRATIGRAPHY OF SITES 834-839, LAU BASIN ${ }^{1}$
}

\author{
Michael J. Styzen ${ }^{2}$
}

\begin{abstract}
Calcareous nannofossils were examined from six sites drilled within the Lau Basin during Ocean Drilling Program Leg 135. These sites lie in a transect roughly west to east across the basin from the Lau Ridge remnant arc to near the active spreading center. The main objective of the biostratigraphic studies was to date the base of the sedimentary section, immediately overlying basement at each site. Material from all these sites yielded calcareous nannofossils. Site 834, which is closest to the Lau Ridge, yielded floras from Holocene to possible late Miocene age. Site 836, furthest from the remnant arc, produced nannofossils of middle Pleistocene age at the base of the sedimentary section. Reworked material was noted from all sites but caused correlation problems only at Site 835 .

One new taxon, Discoaster ono, is described from Pliocene sediments.
\end{abstract}

\section{INTRODUCTION}

Six sites were drilled in the Lau Basin during Ocean Drilling Program (ODP) Leg 135 (Fig. 1). These sites were located in a roughly west to east transect across the basin to provide sample material from the Lau Ridge remnant arc to near the active axial ridge. The sites are all located in north-trending, partially filled, fault-bounded sub-basins. Sites 834 and 835 lie in sub-basins between 100 and $200 \mathrm{~km}$ east of the axis of the Lau Ridge. Sites $836,837,838$, and 839 lie in sub-basins closer to the modern backarc spreading system. The purpose of this part of Leg 135 was to provide information on the history and development of the Lau backarc basin and, by comparison with similar basins, to provide insights into backarc development in general.

Paleontologic studies were conducted mainly to provide dates for the igneous material at the base of the sedimentary sequence at each site.

\section{CALCAREOUS NANNOFOSSIL BIOSTRATIGRAPHY AND GEOCHRONOLOGY}

The biostratigraphic scheme used in this study is that of Bukry (1973, 1975) and Okada and Bukry (1980). The geochronology (Fig. 2) follows mainly that presented in Berggren et al. (1985). Some of the dates have been modified after Gartner (1990). Additional datum horizons that may have some value as correlation points have been added. Most of the datums listed in Table 1 are in general use and require no explanation. Some, however, are based on operational concepts described below.

In this study, Pseudoemiliania lacunosa and Emiliania ovata are considered as distinct species. Perch-Nielsen (1985) regards $E$. ovata as a junior synonym of $P$. lacunos $a$. Considered phylogenetically, this is probably the case as within the range of $P$. lacunosa every intermediate between the two certainly exists. Nannofossil workers in the oil industry of North America generally distinguish two morphotypes, especially near the top of their range. In the Gulf of Mexico, the more delicate circular forms (Plate 1, Figs. 10-11) here called P. lacunosa have a slightly younger extinction than the more robust oval forms (Plate 1, Figs. 8-9), here called E. ovata. This appears also to be the case in Holes 834A and 836A, therefore the two distinct species are retained here.

\footnotetext{
${ }^{1}$ Hawkins, J., Parson, L., Allan, J., et al., 1994. Proc. ODP, Sci. Results, 135: College Station, TX (Ocean Drilling Program).

${ }^{2}$ Shell Offshore Inc., P.O. Box 61933, New Orleans, LA 70161, U.S.A.
}

Gartner (1991) encouraged the documentation of the extinction of Scyphosphaera pulcherrima. That extinction, as noted in this study, is slightly above the one of Helicosphaera sellii.

Gephyrocapsa oceanica, as used in this study, refers to those specimens that have a maximum diameter larger than $3 \mu \mathrm{m}$ and possess a low-angle bridge with respect to the short axis (Plate 2, Fig. 9). Gephyrocapsa caribbeanica, as used in this study, refers to those specimens larger than $3 \mu \mathrm{m}$ that have a very high-angle bridge with respect to the short axis (Plate 2, Fig. 10). With this species concept, the initial occurrence of each of these taxa appear to correspond very well to those used by Bukry in the zonal scheme mentioned above. Gartner (1990) and Shyu and Müller (1991) use a datum based on G. oceanica s.1., with a slightly different species concept for that species as described here; however, the initial occurrence datums for both appear to be similar in age. The extinction event falls approximately at the top of the Olduvai paleomagnetic event, which approximates the Pliocene/ Pleistocene boundary. Using the biostratigraphic framework described above, the $\mathrm{CN} 13 / \mathrm{CN} 14$ boundary would also approximate that age.

\section{METHODS}

Smear slides were prepared for each sample. A small amount of raw material was placed in a small vial with enough distilled water to produce a thin slurry. After thorough mixing with a Vortex Genie, a small drop of the slurry was immediately placed on a clean slide using a disposable glass capillary tube. The slurry was then spread evenly using the side of the capillary tube. The material on the slide was immediately dried on a hot plate. A cover glass was then attached using Piccolyte as a mounting medium. This method of slide preparation provided a uniform layer of material across the entire slide.

Relative abundances were estimated based on counts recorded on a single traverse consisting of 132 fields of view at $980 \times$, each field has a diameter of $220 \mu \mathrm{m}$. Examination of an average slide with a rich flora using this technique takes about $45 \mathrm{~min}$. The following abundance categories were assigned:

$\mathrm{R}=$ rare ( $1-5$ specimens per traverse),

$\mathrm{S}=$ rare to frequent $(6-15$ specimens per traverse),

$\mathrm{F}=$ frequent $(16-35$ specimens per traverse),

$\mathrm{M}=$ frequent to common (36-75 specimens per traverse),

$\mathrm{C}=$ common $(76-150$ specimens per traverse),

$\mathrm{L}=$ common to abundant (151-300 specimens per traverse),

$A=$ abundant (301-600 specimens per traverse),

$\mathrm{V}=$ very abundant $(601-1000$ specimens per traverse $)$,

$\mathrm{W}=$ very very abundant (1001-2000 specimens per traverse), and

$\mathrm{X}=$ extra abundant (over 2000 specimens per traverse). 


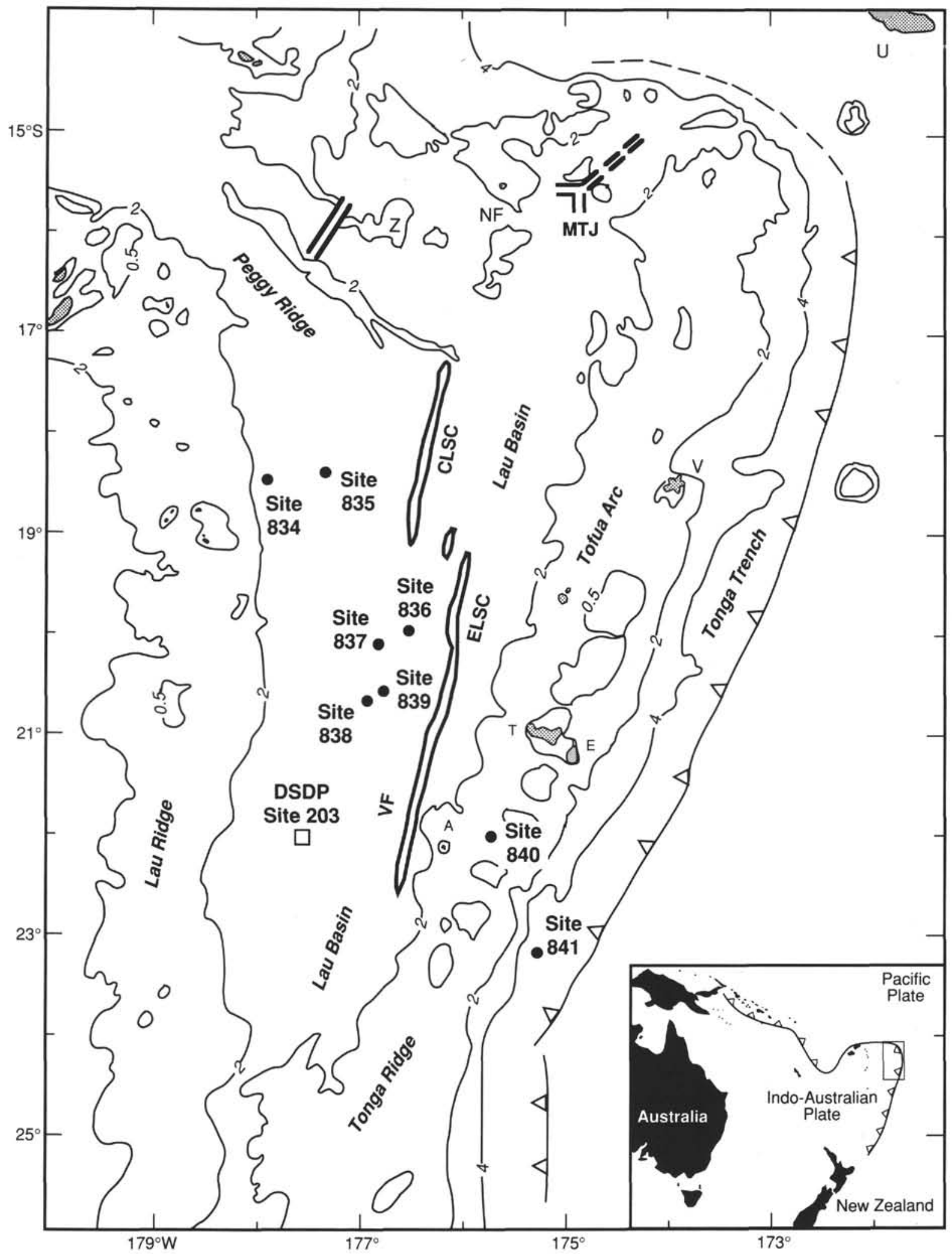

Figure 1. Regional setting for Sites 834 through 839 with the locations of other Leg 135 sites and the major geologic features of the Tonga

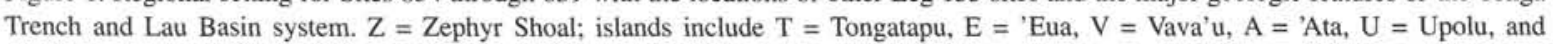
$\mathrm{NF}=$ Niuafo'ou. The locations of the Central Lau (CLSC) and Eastern Lau (ELSC) spreading centers, Valu Fa Ridge (VF), and Mangatolu Triple Junction (MTJ) are shown. The location of DSDP Site 203 is shown as an open box. Contour intervals are in kilometers. (From Parson, Hawkins, Allan, et al., 1992.) 


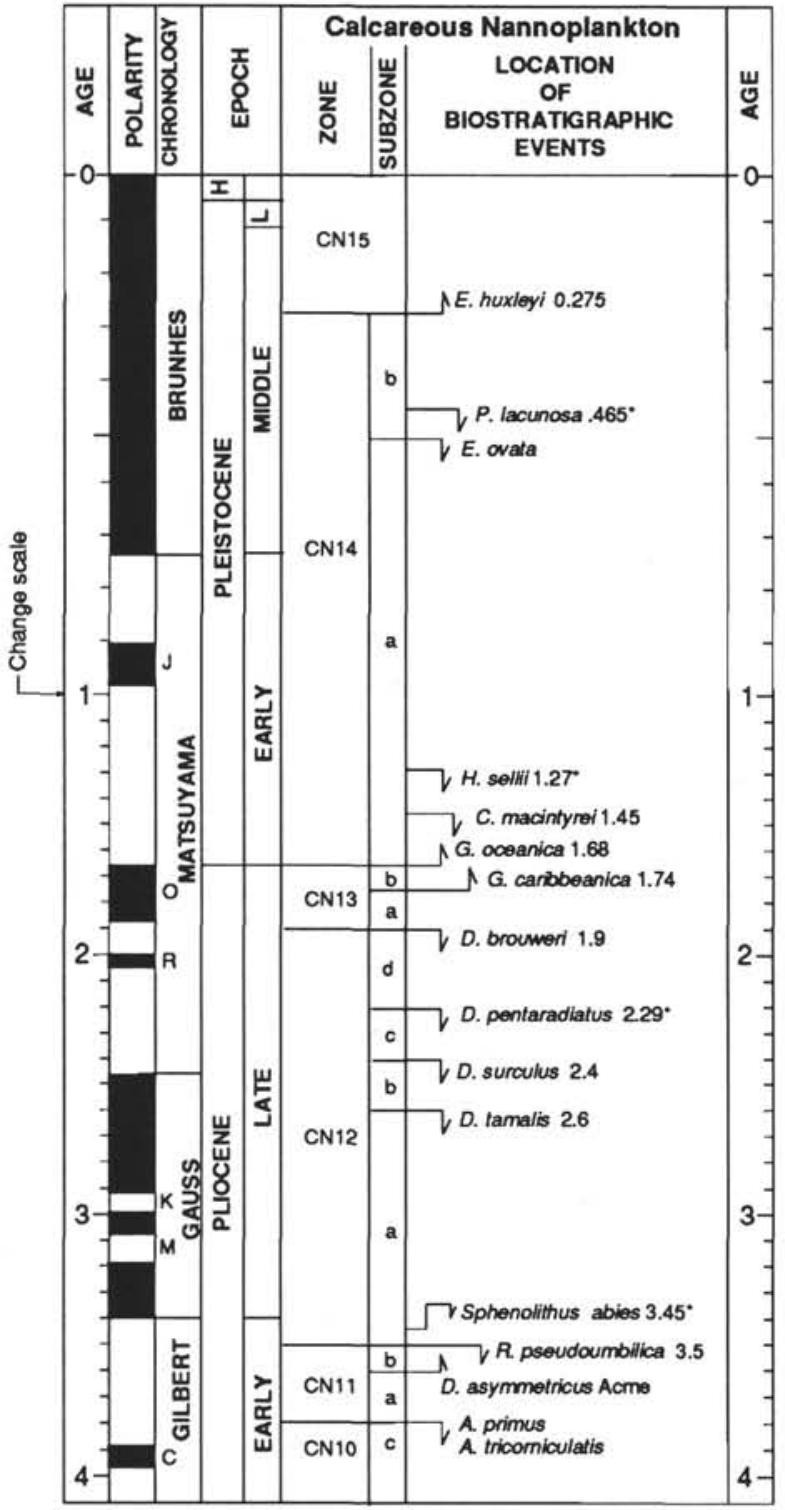

Figure 2. Chronostratigraphic framework adopted for this study. Source of ages of calcareous nannofossil biohorizons are from Berggren et al. (1985), except those marked with an asterisk $\left({ }^{*}\right)$ are from Gartner (1990).
The results were tabulated on an IBM PS/2 using the Bugin data entry system.

\section{SUMMARY OF CALCAREOUS NANNOFOSSIL BIOSTRATIGRAPHY}

\section{Site 834}

Site 834 is located in the western Lau Basin about $100 \mathrm{~km}$ east of the Lau Ridge in a small north-trending sub-basin (Fig. 1). The sedimentary sequence at this site consists of $112.5 \mathrm{~m}$ of clayey nannofossil oozes, turbiditic foraminiferal sands, and oozes and clayey nannofossil mixed sediments. Below 112.5 mbsf, numerous thick basalt flows are interlayered with thin claystone tuffs. The sedimentary sequence was divided into three sedimentary units but these units bear little relation to the nannofossil biostratigraphy. Most of the sedimentary section present at this site was sampled in Hole 834A. The oldest sediment recovered in this hole is probably early Pliocene or slightly older.

A distribution chart of the calcareous nannofossils recovered from Hole $834 \mathrm{~A}$ is given in Table 2. Emiliania huxleyi was present in only the top sample examined, at $2.8 \mathrm{mbsf}$ (Sample 135-834A-1H-1, 130$131 \mathrm{~cm}$ ). Reworking is not evident in this sample. The next datum observed is the highest occurrence of Pseudoemiliania lacunosa at 9.5 mbsf (Sample 135-834A-2H-2, 40-41 cm). This taxon is rare at this depth, but the sample bears a well-preserved and abundant nannofossil flora with no evidence of reworking; therefore, the specimens observed were thought to be in place. This sample also yielded the only specimens of Helicosphaera inversa noted in this hole. The highest observed Emiliania ovata does not occur until the next sample at 22.9 mbsf (Sample 135-834A-3H-4, 134-135 cm). Both $E$. ovata and $P$. lacunosa are very abundant in this sample. As discussed above, these two species are usually lumped under $P$. lacunosa. The distribution and abundance pattern observed here, however, is identical to that observed in the Gulf of Mexico where the two taxa are usually distinguished, at least informally, at their extinction. If the separation observed here represents a widespread occurrence of the temporal separation of the extinction of these two morphotypes, there may be some implications as to the relationship of zonal boundaries in the two widely used nannofossil zonations. The tops of Zone NN19 (Martini, 1971) and Subzone CN14a (Okada and Bukry, 1980) are generally represented as being equivalent in age as in Perch-Nielsen (1985). The top of Zone NN19 is defined as the top of P. lacunosa with no specified morphotype. The top of the Gephyrocapsa oceanica Subzone (=CN14a) as defined by Bukry (1973) is the extinction of $E$. ovata. The fossil distribution as seen here may indicate that the top of Zone NN19 is indeed slightly younger than that of Subzone CN14a.

Table 1. Calcareous nannofossil biohorizons, ages, and depths at Sites 834 through 839 .

\begin{tabular}{|c|c|c|c|c|c|c|c|c|c|c|}
\hline & Biohorizons & $\begin{array}{l}\text { Age } \\
\text { (Ma) }\end{array}$ & $\begin{array}{l}\text { Hole } \\
834 \mathrm{~A}\end{array}$ & $\begin{array}{l}\text { Hole } \\
835 \mathrm{~A}\end{array}$ & $\begin{array}{l}\text { Hole } \\
836 \mathrm{~A}\end{array}$ & $\begin{array}{l}\text { Hole } \\
837 \mathrm{~A}\end{array}$ & $\begin{array}{l}\text { Hole } \\
838 \mathrm{~A}\end{array}$ & $\begin{array}{l}\text { Hole } \\
838 \text { B }\end{array}$ & $\begin{array}{l}\text { Hole } \\
839 \mathrm{~A}\end{array}$ & $\begin{array}{l}\text { Hole } \\
839 B\end{array}$ \\
\hline $\mathrm{HO}$ & Helicosphaera inversa & & 9.5 & & & 2.9 & 3.58 & & 4.5 & \\
\hline LO & Emiliania huxleyi & 0.275 & 2.8 & 0.35 & 0.66 & & 9.2 & & 1.4 & \\
\hline $\mathrm{HO}$ & Pseudoemiliania lacunosa & 0.465 & 9.5 & & 10.7 & 2.9 & 13.2 & & 9.2 & \\
\hline $\mathrm{HO}$ & Emiliania ovata & & 22.9 & & 15.3 & 2.9 & 13.2 & & 9.2 & \\
\hline LO & Helicosphaera inversa & & 2.8 & & & 2.9 & 9.2 & & 9.2 & \\
\hline $\mathrm{HO}$ & Scyphosphaera pulcherrima & & 33.2 & 66.5 & & 41.7 & 29.6 & & 58.4 & \\
\hline $\mathrm{HO}$ & Helicosphaera sellii & 1.27 & 26.6 & & $? 20.2$ & 52.4 & 32.5 & & 86.8 & \\
\hline $\mathrm{HO}$ & Calcidiscus macintyrei & 1.45 & 33.2 & & & & 68.1 & & 138.2 & \\
\hline LO & Gephyrocapsa oceanica & 1.68 & 28.5 & 73.7 & & 63.14 & 53.5 & & 109.2 & \\
\hline LO & Gephyrocapsa caribbeanica & 1.74 & 33.2 & 73.7 & & 70.1 & & & 186.2 & \\
\hline $\mathrm{HO}$ & Discoaster brouweri & 1.9 & 35.3 & & & 80.0 & 91.7 & & & 256.4 \\
\hline $\mathrm{HO}$ & Discoaster pentaradiatus & 2.29 & 35.3 & & & & 91.7 & & & \\
\hline $\mathrm{HO}$ & Discoaster surculus & & 40.5 & & & & & & & \\
\hline $\mathrm{HO}$ & Discoaster tamalis & & 40.5 & & & & & 230.4 & & \\
\hline HO & Sphenolithus neoabies & & 68.0 & & & & & & & \\
\hline $\mathrm{HO}$ & Sphenolithus abies & & 74.2 & & & & & & & \\
\hline $\mathrm{HO}$ & Reticulofenestra pseudoumbilica & & 77.3 & & & & & & & \\
\hline
\end{tabular}

Note: $\mathrm{HO}=$ highest occurrence, and LO $=$ lowest occurrence. 
Table 2. Distribution chart of calcareous nannofossils. Hole 834A.

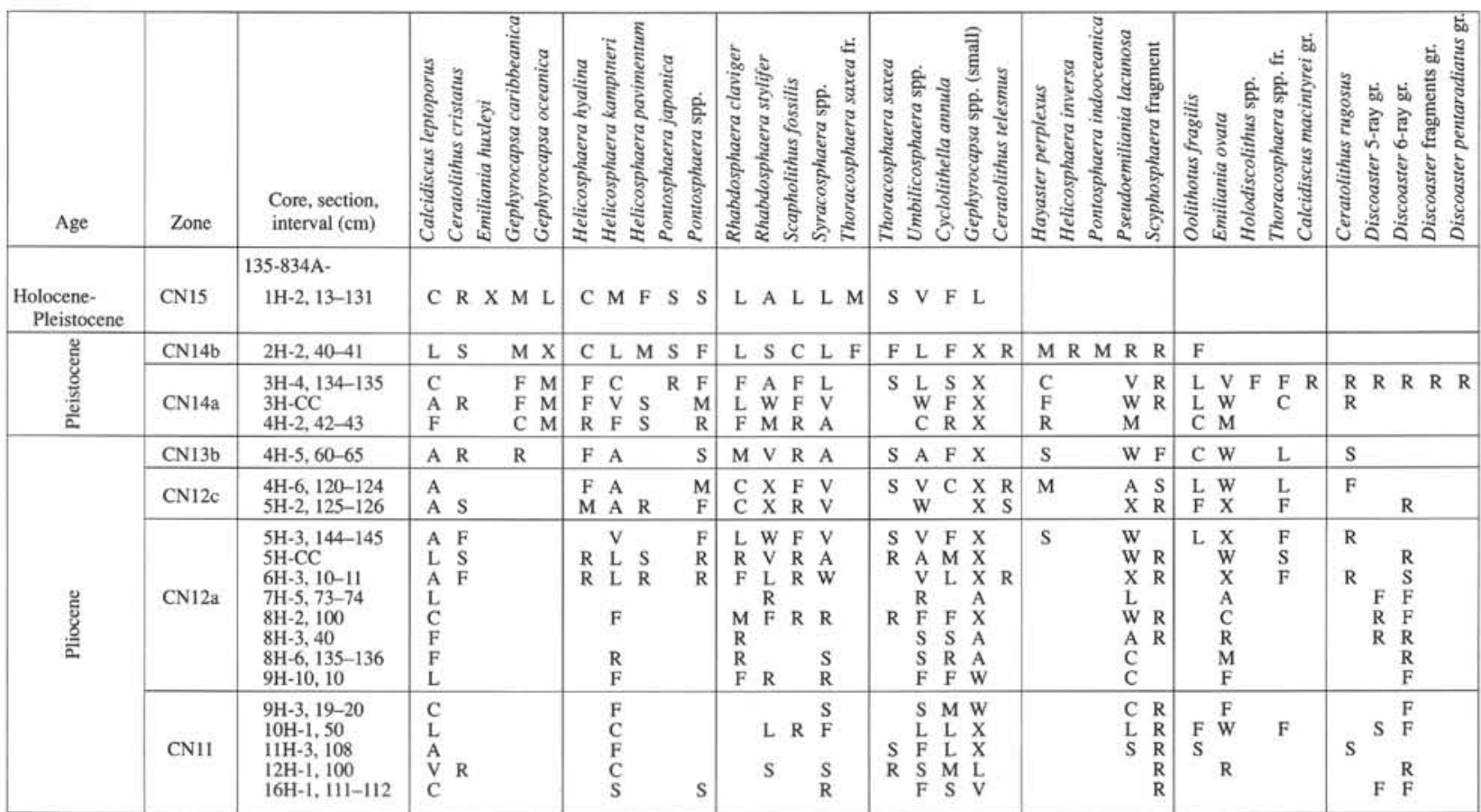

Note: $R=1-5, S=6-15, F=16-35, M=36-75, C=76-150, L=151-300, A=301-600, V=601-1000, W=1001-2000, X=2001+, B=$ taxa absent, gr. $=$ grainy, fr. $=$ fragment.

Sample $135-834 \mathrm{~A}-3 \mathrm{H}-4,134-135 \mathrm{~cm}$, also has some rare, reworked specimens of poorly preserved Pliocene discoasters, sphenoliths and small Reticulofenestra.

The highest observed occurrence of Helicosphaera sellii is at 26.6 mbsf (Sample 135-834A-3H-CC). This sample also shows signs of minor Pliocene reworking. Sample 135-834A-4H-2, 42-43 cm (28.5 mbsf), contains the lowest observed occurrence of Gephyrocapsa oceanica. This datum is the top of Subzone $\mathrm{CN} 13 \mathrm{~b}$, which approximates the Pliocene/Pleistocene boundary. The actual level of this datum is probably between this sample and the next sample examined because the flora here does not include Calcidiscus macintyrei, which has its extinction slightly later than this datum (Fig. 1). At $33.2 \mathrm{mbsf}$ (Sample $135-834 \mathrm{~A}-4 \mathrm{H}-5,60-65 \mathrm{~cm}$ ), the highest observed occurrence of $C$. macintyrei and the lowest observed occurrence of Gephyrocapsa caribbeanica (top of Subzone CN13a) are noted. The highest occurrence in this hole of Scyphosphaera pulcherrima was also observed in this sample; however as it occurs above $H$. sellii in other holes included in this study, this occurrence is probably below its point of extinction. A sample from $35.3 \mathrm{mbsf}$ ( $135-834 \mathrm{~A}-4 \mathrm{H}-6,120-124 \mathrm{~cm}$ ) yielded the highest observed specimens of Discoaster brouweri and $D$. pentaradiatus. The true extinctions of these two species define the top and base of Subzone CN12d, respectively. It is assumed that sediment deposited during that subzone lies between this level and the level of the sample examined immediately above at $33.2 \mathrm{mbsf}$. The single Reticulofenestra pseudoumbilica noted for this sample was assumed to be reworked. A sample at $38.9 \mathrm{mbsf}(135-834 \mathrm{~A}-5 \mathrm{H}-2,125-126 \mathrm{~cm})$ contains a similar flora, but also includes single, poorly preserved, grainy specimens of Discoaster surculus and D. tamalis. These specimens are probably reworked. Sample 135-834A-5H-3, $144 \mathrm{~cm}$ (40.5 mbsf) has a wellpreserved and abundant flora, including common to abundant $D$. surculus and frequent $D$. tamalis. The presence of $D$. tamalis indicates deposition in Subzone CN12a. Subzone CN12b, which is defined on the presence of $D$. surculus without $D$. tamalis, if it is indeed present in this hole, would have to occupy a very short section between 38.9 and 40.5 mbsf. It is possible that Subzone CN12b is missing at this site. The remaining datums present in this hole are the highest observed Sphenolithus neoabies at 68.0 mbsf (Sample 135-834A-8H-3, $40 \mathrm{~cm}$ ), the highest observed Sphenolithus abies at 74 mbsf (Sample 135-
$834 \mathrm{~A}-9 \mathrm{H}-1,10 \mathrm{~cm}$ ), and the highest observed Reticulofenestra pseudoumbilica at 77.3 mbsf (Sample 135-834A-9H-3, 19-20 cm). Samples examined from this sequence contain no obvious reworking. The highest occurrence of $R$. pseudoumbilica defines the top of Zone $\mathrm{CN} 11$. The remaining continuous sedimentary section to the first basalt appears to have been deposited in this zone. Amaurolithus delicatus, present in two samples in this section, may indicate an age as old as Zone CN10. But Amaurolithus tricorniculatus, the zonal marker indicating the top of that zone, was not observed in this hole.

Cycloperfolithus carlae, which previously has been reported only from Miocene sediments (Perch-Nielsen, 1985), is present consistently below 40.5 mbsf.

A sample from sediment interbedded with basalt at $133 \mathrm{mbsf}$ (Sample 135-834A-16X-1, 111-112 cm) contains a moderately preserved, low-diversity flora with taxa characteristic of Zone CN11.

Hole 834B yielded a sample of sediment interbedded with basalt at $312 \mathrm{mbsf}$ (Sample 135-834B-37R-2, 106-107 cm) that appears to be the oldest material recovered in the Lau Basin sites. The flora in this sample is abundant but unfortunately is poorly preserved. The dominant taxa in this flora are Dictyococcites productus, Sphenolithus moriformis, and Dictyococcites perplexa. An extensive search of slides prepared shipboard failed to relocate a single specimen of Triquetrorhabdulus rugosus that was identified for the Initial Reports (Parson, Hawkins, Allan, et al., 1992). It was on the basis of the presence of that taxon that this sample was assigned a late Miocene age in that volume. The flora present contains relatively abundant Dictyococcites and Reticulofenestra and lacks small Gephyrocapsa. Although it is devoid of any other important marker species, this flora is typical of Miocene assemblages. Discoaster deflandrei and Cyclicargolithus floridanus, identified from this sample, are probably reworked. Table 3 is a distribution chart for this sample.

\section{Site 835}

Site 835 is located in the west central Lau Basin, approximately $200 \mathrm{~km}$ east of the remnant arc of the Lau Ridge, and about $80 \mathrm{~km}$ west of the Central Lau Spreading Center (Figure 1). The sedimentary sequence recovered at this site consists of $155 \mathrm{~m}$ of clayey nannofossil 
Table 2 (continued).

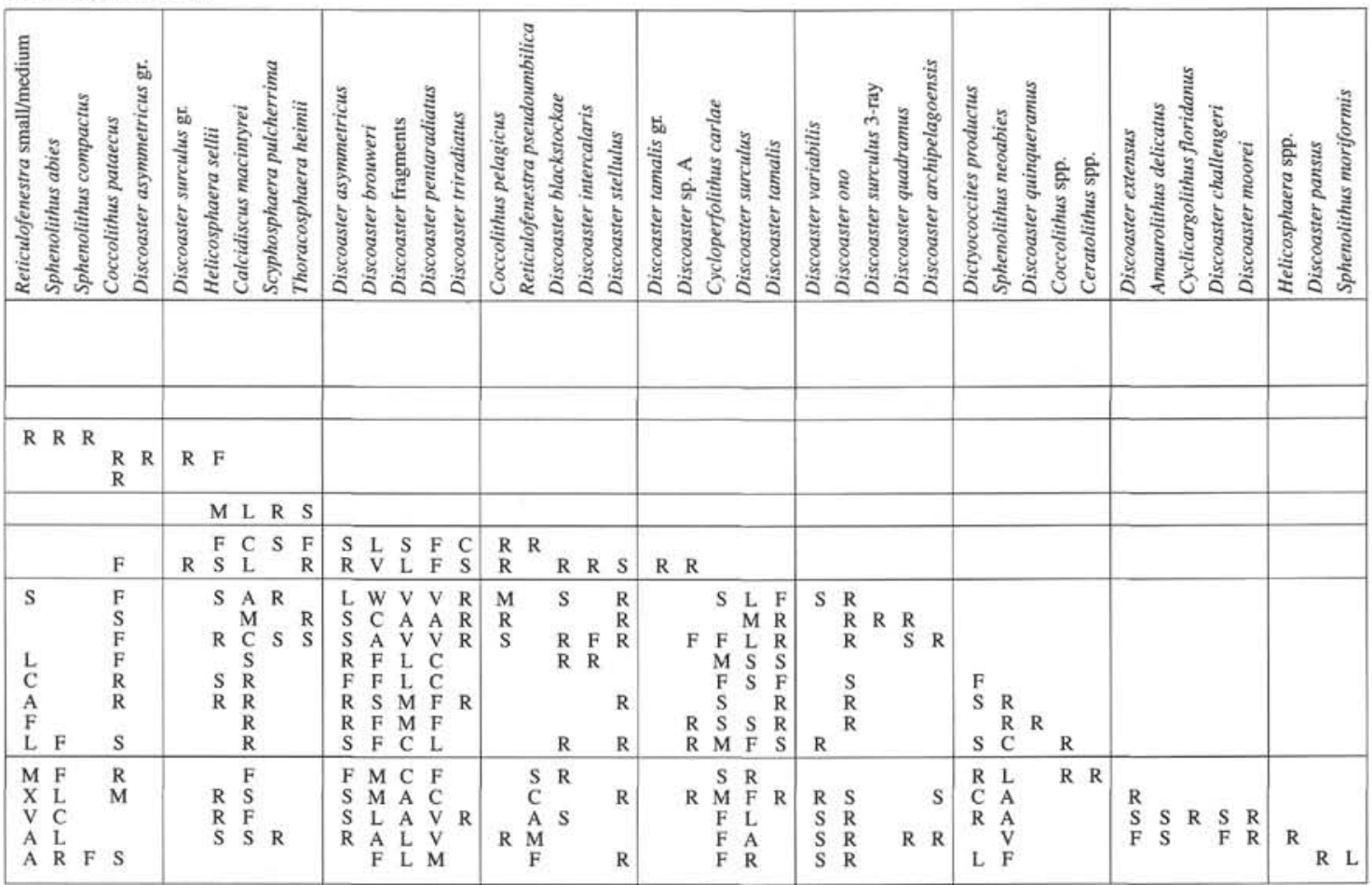

Table 3. Distribution chart of calcareous nannofossils, Hole 834B.

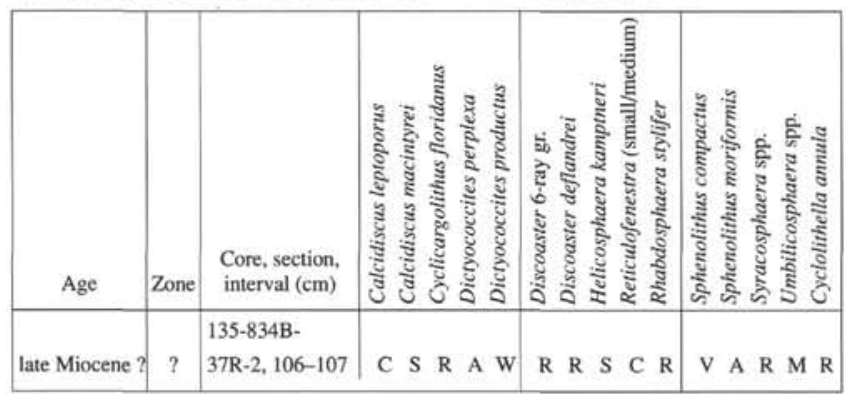

Note: Key as in Table 2.

oozes, mud clast conglomerates, turbiditic foraminiferal sands, and clayey nannofossil mixed sediments. These sediments are often interbedded with epiclastic and volcanic silt layers, especially below 130 mbsf. The sedimentary sequence was divided into two lithologic units, but these units have no relation to nannofossil biostratigraphy.

Sediments recovered from Hole 835A generally contain wellpreserved and abundant calcareous nannofossils, but extensive reworking throughout the section severely limits the biostratigraphic determinations possible. Table 4 is a distribution chart for Hole $835 \mathrm{~A}$.

A sample at $0.35 \mathrm{mbsf}(135-835 \mathrm{~A}-1 \mathrm{H}-1,35-37 \mathrm{~cm})$ yielded an abundant and well-preserved flora, including $E$. huxleyi. This indicates deposition during Subzone CN15. This sample has no sign of reworking. Samples examined from the section between Samples 135-835A-2H-4, 37-38 cm (15.4 mbsf), and 135-835A-8H-5, 120$121 \mathrm{~cm}$ (73.7 mbsf), contain floras that indicate deposition in Zone CN14. All the samples in this interval contain reworked Pliocene floras. A section including parts of Cores $135-835 \mathrm{~A}-7 \mathrm{H}$ and $-8 \mathrm{H}$ yielded samples with no apparent in-place Pleistocene taxa at all. This may be interpreted a rafted block or a thick turbidite unit sampled in those cores. For a more extensive discussion and interpretation of reworking at this site see Rothwell et al. (this volume). The highest observed S. pulcherrima at 66.5 mbsf (Sample 135-835A-7H-7, 55 $\mathrm{cm}$ ) is probably meaningless as it is in a sample with no positively identified in-place flora. With a single exception, the rest of the samples examined to basement contain floras from Zone $\mathrm{CN} 12$ or younger. A sample collected from a mud clast at 124.5 mbsf (135$835 \mathrm{~A}-14 \mathrm{H}-1,100 \mathrm{~cm}$ ) yielded a flora with $S$. abies, S. neoabies, and $R$. pseudoumbilica. This shows that material at least as old as Zone $\mathrm{CN} 11$ is available in the vicinity. No other age determinations were possible at this site.

\section{Site 836}

Site 836 is located about $220 \mathrm{~km}$ west of the Lau Ridge and about $48 \mathrm{~km}$ east of the Eastern Lau Spreading Center. This site is located closest to the active spreading center, and consequently the short ( 23 m) sedimentary sequence overlies the youngest crustal material encountered. The sedimentary sequence comprises a single unit consisting of clayey nannofossil ooze with vitric silt, and clayey vitric mixed sediments. Volcaniclastic material increases down section. Calcareous nannofossils are present, abundant, and generally well-preserved throughout the section. The oldest sediment recovered at this site is early Pleistocene in age.

Table 5 is a distribution chart of calcareous nannofossils observed in Hole 836A. A sample at $0.66 \mathrm{mbsf}(135-836 \mathrm{~A}-1 \mathrm{H}-1,66-67 \mathrm{~cm})$ contains an assemblage that includes E. huxleyi. This indicates that Holocene sediment (Zone CN15) is present at this depth. Two samples examined between this Holocene material and the next datum contain neither E. huxleyi nor E. ovata; that section, therefore, is assigned to Subzone CN14b. The highest observed occurrence of $P$. lacunosa was at $10.7 \mathrm{mbsf}$ (Sample 135-836A-2H-CC). The highest observed occurrence of $E$. ovata was at 15.3 mbsf (Sample 135-836A-3H-4, $10-11 \mathrm{~cm}$ ). This stratigraphic relationship of these two taxa is similar to that observed in Hole 834A. The remaining possible datum from this hole is the highest observed $H$. sellii. Only a single specimen of this taxon was identified from Sample 135-836A-3H-CC (20.2 mbsf), but this sample also contains Pliocene reworked material so this datum is questionable. In any case, no in-place flora older than Subzone $\mathrm{CN} 14 \mathrm{~A}$ was identified at this site.

\section{Site 837}

Site 837 is located in the central Lau Basin about $160 \mathrm{~km}$ east of the Lau Ridge remnant arc and approximately $69 \mathrm{~km}$ west of the axial 
Table 4. Distribution chart of calcareous nannofossils, Hole 835A.

\begin{tabular}{|c|c|c|c|c|c|c|c|c|c|c|c|c|c|c|c|c|c|c|c|c|c|c|c|c|c|c|}
\hline Age & Zone & $\begin{array}{l}\text { Core, section, } \\
\text { interval }(\mathrm{cm})\end{array}$ & 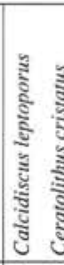 & 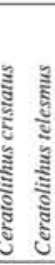 & 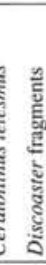 & 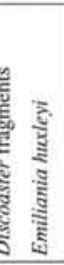 & 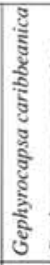 & 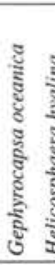 & 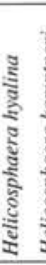 & 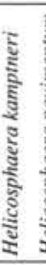 & 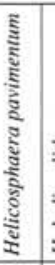 & 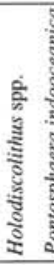 & 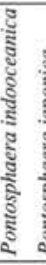 & 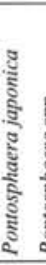 & 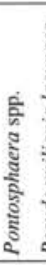 & 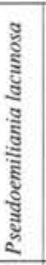 & 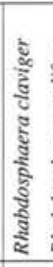 & 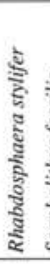 & 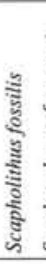 & 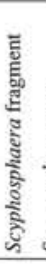 & 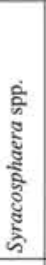 & 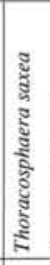 & $\frac{5}{3}$ & 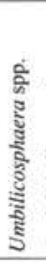 & 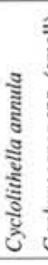 & 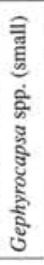 \\
\hline $\begin{array}{l}\text { Holocene- } \\
\text { Pleistocene }\end{array}$ & $\mathrm{CN} 15$ & $\begin{array}{l}135-835 \mathrm{~A}- \\
1 \mathrm{H}-1,35-37\end{array}$ & L & S R & R F & R X & M & C & C & C & M & $\mathbf{R}$ & R & $\mathrm{S}$ & R & R & L & L & C & $\mathrm{R}$ & L & C & $\mathrm{R}$ & L & M & W \\
\hline$\frac{\frac{\pi}{8}}{\frac{g}{n}}$ & $\mathrm{CN} 14$ & $\begin{array}{l}2 \mathrm{H}-4,37-38 \\
3 \mathrm{H}-7,18-19 \\
4 \mathrm{H}-5,52-53 \\
5 \mathrm{H}-7,10-11 \\
6 \mathrm{H}-3,30 \\
6 \mathrm{H}-4,60 \\
6 \mathrm{H}-5,30 \\
6 \mathrm{H}-6,60 \\
6 \mathrm{H}-6,90 \\
6 \mathrm{H}-6,120 \\
7 \mathrm{H}-1,30 \\
7 \mathrm{H}-2,40 \\
7 \mathrm{H}-3,70 \\
7 \mathrm{H}-4,90 \\
7 \mathrm{H}-5,60\end{array}$ & $\begin{array}{l}\text { M } \\
\text { C } \\
\text { L } \\
\text { R } \\
\text { C } \\
\text { C } \\
\text { L } \\
\text { L } \\
\text { L } \\
\text { A } \\
\text { A } \\
\text { A } \\
\text { L } \\
\text { C } \\
\text { C }\end{array}$ & $\begin{array}{l}\mathrm{R} \\
\mathrm{R} \\
\mathrm{S}\end{array}$ & $\begin{array}{r}\mathrm{R} \\
\mathrm{F} \\
\mathrm{F} \\
\mathrm{I} \\
\mathrm{I}\end{array}$ & $\begin{array}{l}\text { A } \\
\text { R } \\
\text { L } \\
\text { F } \\
\text { S } \\
\text { C }\end{array}$ & $\begin{array}{l}\text { F } \\
\text { R } \\
\text { R } \\
\text { R } \\
\text { F } \\
\text { A } \\
\text { S } \\
\text { S } \\
\text { S }\end{array}$ & $\begin{array}{l}\mathrm{M} \\
\mathrm{F} \\
\mathrm{S} \\
\mathrm{C} \\
\mathrm{M} \\
\mathrm{L} \\
\mathrm{R} \\
\mathrm{A} \\
\mathrm{C} \\
\mathrm{R}\end{array}$ & $\begin{array}{l}\text { R } \\
\text { F } \\
M \\
\text { L } \\
\text { S } \\
\text { F } \\
\text { M } \\
\text { F } \\
\text { M } \\
\text { S } \\
\text { F } \\
\text { F }\end{array}$ & $\begin{array}{l}\text { M } \\
\text { S } \\
\text { C } \\
\text { R } \\
\text { C } \\
\text { M } \\
\text { L } \\
\text { L } \\
\text { A } \\
\text { A } \\
\text { L } \\
\text { L } \\
\text { C } \\
\text { C } \\
\text { C }\end{array}$ & $\begin{array}{l}\mathrm{R} \\
\mathrm{S} \\
\mathrm{R}\end{array}$ & R & & & $\begin{array}{l}\mathrm{R} \\
\mathrm{R} \\
\\
\mathrm{F} \\
\mathrm{F} \\
\mathrm{R} \\
\\
\mathrm{R} \\
\mathrm{S} \\
\mathrm{S} \\
\mathrm{R} \\
\mathrm{R}\end{array}$ & $\begin{array}{l}\text { V } \\
\text { C } \\
\text { V } \\
\text { R } \\
\text { L } \\
\text { L } \\
\text { X } \\
\text { W } \\
\text { X } \\
\text { W } \\
\text { V } \\
\text { W } \\
\text { W } \\
\text { V } \\
\text { V }\end{array}$ & $\begin{array}{l}\text { R } \\
\text { F } \\
\text { S } \\
\text { R } \\
\text { C } \\
\text { S } \\
\text { L } \\
\text { A } \\
\text { F } \\
\text { M } \\
\text { M } \\
\text { A } \\
\text { C } \\
\text { C } \\
\text { C }\end{array}$ & $\begin{array}{l}\text { M } \\
\text { F } \\
\text { S } \\
\text { R } \\
\text { M } \\
\text { F } \\
\text { L } \\
\text { A } \\
\text { V } \\
\text { V } \\
\text { C } \\
\text { A } \\
\text { A } \\
\text { L } \\
\text { A }\end{array}$ & $\begin{array}{l}\mathrm{R} \\
\mathrm{F} \\
\mathrm{F} \\
\mathrm{F} \\
\mathrm{R} \\
\mathrm{R} \\
\mathrm{S} \\
\mathrm{S} \\
\mathrm{S} \\
\mathrm{F}\end{array}$ & $\begin{array}{l}\mathrm{R} \\
\mathrm{R} \\
\mathrm{F} \\
\mathrm{R} \\
\mathrm{R} \\
\mathrm{F} \\
\mathrm{R} \\
\mathrm{R} \\
\mathrm{S}\end{array}$ & $\begin{array}{l}\mathrm{F} \\
\mathrm{M} \\
\mathrm{C} \\
\mathrm{S} \\
\mathrm{V} \\
\mathrm{C} \\
\mathrm{L} \\
\mathrm{A} \\
\mathrm{V} \\
\mathrm{A} \\
\mathrm{A} \\
\mathrm{A} \\
\mathrm{L} \\
\mathrm{C} \\
\mathrm{L}\end{array}$ & $\begin{array}{l}\mathrm{S} \\
\\
\mathrm{S} \\
\mathrm{S} \\
\\
\mathrm{R} \\
\mathrm{R}\end{array}$ & $\begin{array}{l}\mathrm{F} \\
\mathrm{S} \\
\mathrm{M} \\
\mathrm{F} \\
\mathrm{F} \\
\mathrm{S} \\
\mathrm{C} \\
\mathrm{M} \\
\mathrm{M} \\
\mathrm{M} \\
\mathrm{F} \\
\mathrm{S} \\
\mathrm{S}\end{array}$ & $\begin{array}{l}\text { C } \\
\text { S } \\
\text { A } \\
\text { L } \\
\text { A } \\
\text { L } \\
\text { L } \\
\text { V } \\
\text { A } \\
\text { A } \\
\text { L } \\
\text { L } \\
\text { A }\end{array}$ & $\begin{array}{l}\mathrm{M} \\
\mathrm{R} \\
\mathrm{F} \\
\mathrm{F} \\
\mathrm{F} \\
\mathrm{M} \\
\mathrm{S} \\
\mathrm{R} \\
\mathrm{F} \\
\mathrm{M} \\
\mathrm{S} \\
\mathrm{S} \\
\mathrm{S} \\
\mathrm{R}\end{array}$ & $\begin{array}{l}\text { W } \\
\text { W } \\
\text { X } \\
\text { V } \\
\text { X } \\
\text { X } \\
\text { X } \\
\text { X } \\
\text { X } \\
\text { X } \\
\text { X } \\
\text { X } \\
\text { X } \\
\text { X }\end{array}$ \\
\hline 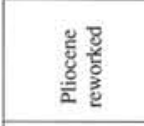 & \multirow{2}{*}{$\mathrm{CN} 12$} & $\begin{array}{l}7 \mathrm{H}-6,90 \\
7 \mathrm{H}-7,55 \\
8 \mathrm{H}-2,54 \\
8 \mathrm{H}-2,100 \\
8 \mathrm{H}-2,120-121\end{array}$ & $\begin{array}{l}\mathrm{L} \\
\mathrm{L} \\
\mathrm{L} \\
\mathrm{C} \\
\mathrm{M}\end{array}$ & $\begin{array}{l}\mathrm{S} \\
\mathrm{R}\end{array}$ & $\begin{array}{ll}1 \\
\text { R }\end{array}$ & $\begin{array}{l}\mathrm{M} \\
\mathrm{C} \\
\mathrm{W} \\
\mathrm{A} \\
\mathrm{C}\end{array}$ & & & $\begin{array}{l}\mathrm{F} \\
\mathrm{R} \\
\mathrm{R} \\
\mathrm{R}\end{array}$ & $\begin{array}{l}\mathrm{L} \\
\mathrm{C} \\
\mathrm{L} \\
\mathrm{L} \\
\mathrm{F}\end{array}$ & & \multirow[b]{2}{*}{$\begin{array}{l}\mathrm{R} \\
\mathrm{R} \\
\mathrm{R}\end{array}$} & & & $\begin{array}{l}\mathrm{S} \\
\mathrm{R} \\
\mathrm{R} \\
\mathrm{S}\end{array}$ & $\begin{array}{c}\mathrm{V} \\
\mathrm{W} \\
\mathrm{X} \\
\mathrm{W} \\
\mathrm{A}\end{array}$ & $\begin{array}{l}\mathrm{F} \\
\mathrm{F} \\
\mathrm{F} \\
\mathrm{S}\end{array}$ & $\begin{array}{l}\text { V } \\
\text { V } \\
\text { V } \\
\text { L } \\
\text { F }\end{array}$ & $\begin{array}{l}\mathrm{F} \\
\mathrm{R} \\
\mathrm{R}\end{array}$ & $\begin{array}{l}\mathrm{R} \\
\mathrm{R} \\
\mathrm{F}\end{array}$ & $\begin{array}{l}\mathrm{A} \\
\mathrm{L} \\
\mathrm{A} \\
\mathrm{A} \\
\mathrm{C}\end{array}$ & \multirow[b]{2}{*}{$\mathrm{R}$} & $\begin{array}{l}\mathrm{M} \\
\mathrm{F} \\
\mathrm{S} \\
\mathrm{F}\end{array}$ & $\begin{array}{l}\mathrm{A} \\
\mathrm{A} \\
\mathrm{V} \\
\mathrm{F} \\
\mathrm{F}\end{array}$ & $\begin{array}{l}\text { S } \\
\text { R } \\
\text { A } \\
\text { L } \\
\text { F }\end{array}$ & $\begin{array}{l}\mathrm{X} \\
\mathrm{X} \\
\mathrm{X} \\
\mathrm{X} \\
\mathrm{W}\end{array}$ \\
\hline$\frac{\frac{d}{8}}{\frac{d}{\pi}}$ & & $\begin{array}{l}8 \mathrm{H}-2,144 \\
8 \mathrm{H}-2,145 \\
8 \mathrm{H}-3,58 \\
8 \mathrm{H}-5,120\end{array}$ & $\begin{array}{l}\mathrm{L} \\
\mathrm{L} \\
\mathrm{C} \\
\mathrm{L}\end{array}$ & $\begin{array}{l}\mathrm{R} \\
\mathrm{R}\end{array}$ & & $\begin{array}{l}\mathrm{C} \\
\mathrm{C} \\
\mathrm{F}\end{array}$ & $\begin{array}{l}\mathrm{M} \\
\mathrm{F} \\
\mathrm{F} \\
\mathrm{M}\end{array}$ & $\begin{array}{l}\mathrm{M} \\
\mathrm{F} \\
\mathrm{F} \\
\mathrm{F}\end{array}$ & $\begin{array}{l}\mathrm{C} \\
\mathrm{M} \\
\mathrm{M} \\
\mathrm{F}\end{array}$ & $\begin{array}{l}\mathrm{L} \\
\mathrm{L} \\
\mathrm{M} \\
\mathrm{L}\end{array}$ & & & & $\begin{array}{l}\mathrm{R} \\
\mathrm{R}\end{array}$ & $\begin{array}{l}\mathrm{F} \\
\mathrm{R} \\
\mathrm{S} \\
\mathrm{F}\end{array}$ & $\begin{array}{l}\text { V } \\
\text { W } \\
\text { L } \\
\text { W }\end{array}$ & $\begin{array}{l}\text { C } \\
\text { C } \\
\text { C } \\
\text { A }\end{array}$ & $\begin{array}{l}\mathrm{A} \\
\mathrm{A} \\
\mathrm{C} \\
\mathrm{A}\end{array}$ & $\begin{array}{l}\mathrm{S} \\
\mathrm{F} \\
\mathrm{R} \\
\mathrm{S}\end{array}$ & $\begin{array}{l}\mathrm{S} \\
\mathrm{R} \\
\mathrm{S} \\
\mathrm{F}\end{array}$ & $\begin{array}{l}C \\
C \\
C \\
L\end{array}$ & & $\begin{array}{l}\mathrm{F} \\
\mathrm{F} \\
\mathrm{F} \\
\mathrm{M}\end{array}$ & $\begin{array}{l}\mathrm{L} \\
\mathrm{A} \\
\mathrm{C} \\
\mathrm{L}\end{array}$ & $\begin{array}{l}\mathrm{F} \\
\mathrm{F} \\
\mathrm{F} \\
\mathrm{S}\end{array}$ & $\begin{array}{l}\mathrm{X} \\
\mathrm{X} \\
\mathrm{X} \\
\mathrm{X}\end{array}$ \\
\hline \multirow{3}{*}{$\begin{array}{l}\text { हूँ } \\
\frac{\delta}{2} \\
\end{array}$} & $\mathrm{CN} 12$ & $\begin{array}{l}9 \mathrm{H}-5,70-71 \\
10 \mathrm{H}-5,41-42 \\
11 \mathrm{H}-5,88-89 \\
12 \mathrm{H}-3,72-73 \\
13 \mathrm{H}-4,125-126\end{array}$ & $\begin{array}{l}\text { C } \\
\text { A } \\
\text { L } \\
\text { L } \\
\text { C }\end{array}$ & $\begin{array}{l}\mathrm{R} \\
\mathrm{S}\end{array}$ & & $\begin{array}{l}\mathrm{C} \\
\mathrm{M} \\
\mathrm{V} \\
\mathrm{V} \\
\mathrm{C}\end{array}$ & & & $\begin{array}{l}\mathrm{S} \\
\mathrm{F} \\
\mathrm{R}\end{array}$ & $\begin{array}{l}\mathrm{L} \\
\mathrm{A} \\
\mathrm{L} \\
\mathrm{C} \\
\mathrm{F}\end{array}$ & & $\mathbf{R}$ & & & $\begin{array}{l}\mathrm{S} \\
\mathrm{S} \\
\mathrm{S} \\
\mathrm{S}\end{array}$ & $\begin{array}{l}\text { A } \\
\text { W } \\
\text { W } \\
\text { W } \\
\text { A }\end{array}$ & $\begin{array}{l}\mathrm{F} \\
\mathrm{L} \\
\mathrm{F} \\
\mathrm{F} \\
\mathrm{F}\end{array}$ & $\begin{array}{l}\text { A } \\
\text { W } \\
\text { L } \\
\text { L } \\
\text { C }\end{array}$ & $\begin{array}{l}\mathrm{S} \\
\mathrm{F} \\
\mathrm{R} \\
\mathrm{R}\end{array}$ & $\begin{array}{l}\mathrm{S} \\
\mathrm{R} \\
\mathrm{F} \\
\mathrm{R} \\
\mathrm{R}\end{array}$ & $\begin{array}{l}\mathrm{L} \\
\mathrm{A} \\
\mathrm{A} \\
\mathrm{L} \\
\mathrm{M}\end{array}$ & & $\begin{array}{l}\mathrm{F} \\
\mathrm{F} \\
\mathrm{F} \\
\mathrm{R} \\
\mathrm{F}\end{array}$ & $\begin{array}{l}\mathrm{L} \\
\mathrm{V} \\
\mathrm{A} \\
\mathrm{A} \\
\mathrm{C}\end{array}$ & $\begin{array}{l}\mathrm{S} \\
\mathrm{S} \\
\mathrm{F} \\
\mathrm{F} \\
\mathrm{F}\end{array}$ & $\begin{array}{l}\mathrm{X} \\
\mathrm{X} \\
\mathrm{X} \\
\mathrm{X} \\
\mathrm{W}\end{array}$ \\
\hline & CN11 & $14 \mathrm{H}-1,100-101$ & $\mathrm{C}$ & $\mathbf{R}$ & & $\mathrm{A}$ & & & & $\mathrm{F}$ & & & & & $\mathrm{S}$ & $\mathrm{A}$ & $\mathrm{s}$ & $\mathrm{M}$ & & $\mathrm{R}$ & $\mathrm{F}$ & $s$ & $\mathrm{~A}$ & $\mathrm{~V}$ & $\mathrm{~A}$ & $\mathrm{~A}$ \\
\hline & $\mathrm{CN} 12$ & $\begin{array}{l}14 \mathrm{H}-7,31 \\
15 \mathrm{H}-5,140-141 \\
16 \mathrm{H}-4,129-130 \\
18 \mathrm{X}-1,1-2\end{array}$ & $\begin{array}{l}\mathrm{L} \\
\mathrm{M} \\
\mathrm{C} \\
\mathrm{C}\end{array}$ & $\begin{array}{l}\mathrm{F} \\
\mathrm{R}\end{array}$ & & $\begin{array}{l}\text { W } \\
\text { A } \\
\text { V } \\
\text { W }\end{array}$ & & & $\mathrm{R}$ & $\begin{array}{l}\mathrm{A} \\
\mathrm{R} \\
\mathrm{S}\end{array}$ & & & & & $\mathrm{S}$ & $\begin{array}{l}\mathrm{W} \\
\mathrm{V} \\
\mathrm{C} \\
\mathrm{V}\end{array}$ & $\begin{array}{l}\mathrm{S} \\
\mathrm{R} \\
\mathrm{F}\end{array}$ & $\begin{array}{l}\mathrm{V} \\
\mathrm{R}\end{array}$ & $\mathbf{R}$ & $\mathrm{S}$ & $\begin{array}{c}\mathrm{A} \\
\mathrm{M} \\
\mathrm{F} \\
\mathrm{F}\end{array}$ & & $\begin{array}{l}\mathrm{S} \\
\mathrm{R} \\
\mathrm{S}\end{array}$ & $\begin{array}{l}\mathrm{V} \\
\mathrm{S} \\
\mathrm{F}\end{array}$ & $\begin{array}{l}\mathrm{F} \\
\mathrm{F} \\
\mathrm{S} \\
\mathrm{M}\end{array}$ & $\begin{array}{l}\mathrm{X} \\
\mathrm{W} \\
\mathrm{V} \\
\mathrm{A}\end{array}$ \\
\hline
\end{tabular}

rift zone of the Eastern Lau Spreading center (Fig. 1). The sedimentary sequence recovered at this site consists of $84 \mathrm{~m}$ of clayey nannofossil oozes and volcaniclastic turbidites. The sequence is subdivided into two lithologic units that have no relationship to biostratigraphic subdivisions. The oldest sediment recovered at this site is late Pliocene in age. Calcareous nannofossils are generally abundant and well preserved, but both preservation and abundance decrease downhole. Reworking of Pliocene taxa in Pleistocene turbidites is present but not to the extent of the reworking at Site 835 .

Table 6 is a distribution chart of calcareous nannofossils recovered in Hole 837A. The flora from a sample from $2.9 \mathrm{mbsf}$ (Sample 135-837A-1H-2, 104-105 cm) contains $P$. lacunosa, $E$. ovata, and Helicosphaera inversa. This sample is assigned to Subzone CN14a. The next two datums encountered in this hole also lie within Subzone $\mathrm{CN} 14 \mathrm{a}$. They are the highest observed $S$. pulcherrima at $41.7 \mathrm{mbsf}$ (Sample 135-837A-5H-4, 123-124 cm) and the highest observed $H$. sellii at 52.4 mbsf (Sample 135-837A-6H-5, 38-39 cm). The single specimen of $H$. sellii identified from Sample 135-837A-1H-CC was considered reworked as rich floras immediately below do not contain individuals of that taxon. The extinction of $C$. macintyrei, which also falls in Subzone CN14A, is not picked in this hole as rare individuals occur sporadically throughout the upper part of this hole associated with rare reworked discoasters. $C$. macintyrei does not occur consistently in this hole until within the Pliocene section. The lowest observed occurrence of G. oceanica is at $63.14 \mathrm{mbsf}$ (Sample 135-837A-7H-6, $14-15 \mathrm{~cm})$. That of G. caribbeanica is in Sample 135-837A-8H-4, $109-110 \mathrm{~cm}$ (70.1 mbsf). The section with floras containing G. caribbeanica but not $G$. oceanica represents deposition in Subzone CN13b. Rare $D$. brouweri occur in reworked floras throughout the section, but the abundance of that taxon greatly increases in Sample 135-837A-9H-
$4,99-100 \mathrm{~cm}$ ( $80 \mathrm{mbsf})$. The datum based on the highest occurrence of $D$. brouweri (top Subzone CN12a) is placed at this depth.

\section{Site 838}

Site 838 is located in the central Lau basin about $87 \mathrm{~km}$ west of the Central Lau Spreading Center and approximately $125 \mathrm{~km}$ east of the axis of the Lau Ridge remnant arc (Fig. 1). The sedimentary sequence penetrated at this site is $103.2 \mathrm{~m}$ thick. Sediments were divided into three lithologic units. Unit $\mathrm{I}$, which includes sediments to 23.4 mbsf, consists of clayey nannofossil oozes and thin interbedded volcaniclastic turbidites. Recovery was good in this unit, and the lithology was conducive to nannofossil preservation. Floras recovered from Unit I are abundant and well preserved. Unit II is an upward-fining sequence with coarse volcaniclastic gravels at its base and vitric silts with interbedded clayey nannofossil oozes at the top. This unit extends from the base of Unit I to $98.7 \mathrm{mbsf}$. The upper part of this unit yielded good nannofossil floras. Most lithologies in the lower part of this unit, however, are too coarse to be conducive to the accumulation and preservation of calcareous nannofossils so the floras recovered from them tend to be spotty, concentrated in whatever thin, fine-grained layers are present. Unit III (98.7-259.2 mbsf) had spotty, very poor recovery. Sediments from this unit, which include pumiceous volcanic gravel, volcanic conglomerate, vitric sandstone, and vitric clayey siltstone, sometimes yielded excellent, well-preserved floras; however, the record is very discontinuous. Given the lithology and the poor recovery, one should consider any biostratigraphic determinations below Core $135-838 \mathrm{~A}-7 \mathrm{H}$ to be spot information rather than part of a recognizable sequence. The oldest sediments encountered at this site are late Pliocene in age. 


\begin{tabular}{|c|c|c|c|c|c|c|c|c|c|c|c|c|c|c|c|c|c|c|c|c|c|c|c|c|c|c|c|c|c|c|c|c|c|}
\hline 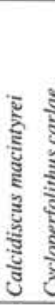 & 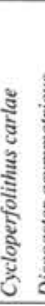 & 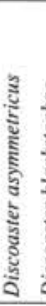 & 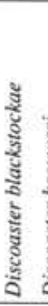 & 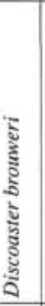 & 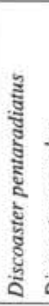 & 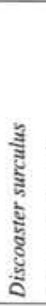 & 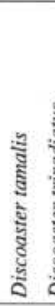 & 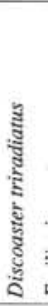 & 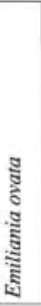 & 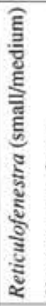 & & 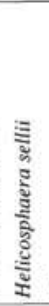 & 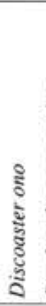 & 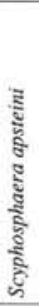 & 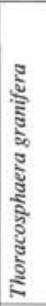 & 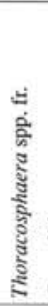 & . & 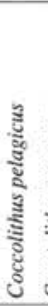 & 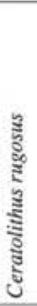 & 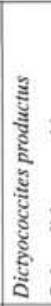 & 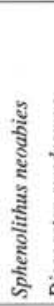 & 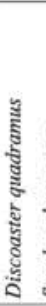 & 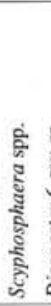 & 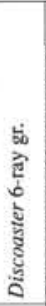 & 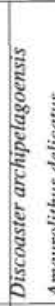 & 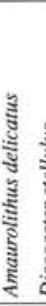 & 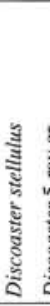 & 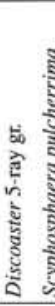 & 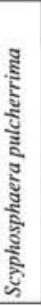 & 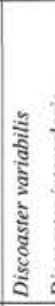 & 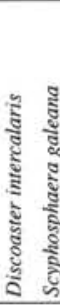 & 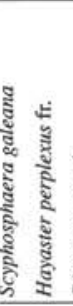 & 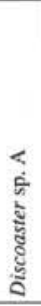 \\
\hline $\begin{array}{l} \\
\mathrm{S} \\
\mathrm{C} \\
\mathrm{C} \\
\mathrm{F} \\
\mathrm{M} \\
\mathrm{S} \\
\mathrm{S}\end{array}$ & $\begin{array}{l}\mathrm{F} \\
\mathrm{R} \\
\mathrm{S}\end{array}$ & $\begin{array}{l}\mathrm{F} \\
\mathrm{R} \\
\mathrm{R}\end{array}$ & F & $\begin{array}{l}\mathrm{A} \\
\mathrm{R} \\
\mathrm{A} \\
\\
\mathrm{R} \\
\mathrm{L} \\
\mathrm{R} \\
\mathrm{R} \\
\mathrm{L} \\
\mathrm{R} \\
\mathrm{L} \\
\mathrm{L} \\
\mathrm{A}\end{array}$ & $\begin{array}{l}\text { W } \\
\text { F } \\
\text { W } \\
\text { R } \\
\text { R } \\
\text { L } \\
\text { R } \\
\text { R } \\
\text { L } \\
\text { R } \\
\text { S } \\
\text { R } \\
\text { R }\end{array}$ & $\begin{array}{l}\mathrm{C} \\
\mathrm{R} \\
\mathrm{M}\end{array}$ & $\begin{array}{l}\mathrm{R} \\
\mathrm{R} \\
\mathrm{F} \\
\\
\mathrm{R}\end{array}$ & $\begin{array}{c}\mathrm{R} \\
\mathrm{R} \\
\\
\\
\mathrm{R} \\
\mathrm{R} \\
\mathrm{M} \\
\mathrm{F} \\
\mathrm{F}\end{array}$ & $\begin{array}{l}\text { A } \\
\text { L } \\
\text { V } \\
\text { M } \\
\text { C } \\
\text { V } \\
\text { W } \\
\text { W } \\
\text { V } \\
\text { V } \\
\text { W } \\
\text { W } \\
\text { V } \\
\text { W }\end{array}$ & $\begin{array}{l}\mathrm{L} \\
\mathrm{S} \\
\mathrm{L} \\
\\
\mathrm{R} \\
\mathrm{R} \\
\mathrm{F} \\
\mathrm{R} \\
\mathrm{R} \\
\mathrm{S}\end{array}$ & $\begin{array}{l}\mathrm{F} \\
\mathrm{R} \\
\mathrm{F} \\
\mathrm{S} \\
\mathrm{F} \\
\mathrm{S} \\
\mathrm{F} \\
\mathrm{F} \\
\mathrm{S} \\
\mathrm{F} \\
\mathrm{R} \\
\mathrm{R}\end{array}$ & $\begin{array}{l}\mathrm{S} \\
\mathrm{F} \\
\mathrm{F} \\
\mathrm{F} \\
\mathrm{F} \\
\mathrm{F} \\
\mathrm{R} \\
\mathrm{S}\end{array}$ & $R$ & $\mathbf{R}$ & $\mathbf{R}$ & $\begin{array}{l}\mathrm{M} \\
\mathrm{F} \\
\mathrm{M} \\
\mathrm{F} \\
\mathrm{F} \\
\mathrm{F} \\
\mathrm{R}\end{array}$ & R & $\mathrm{R}$ & $\mathbf{R}$ & R & R & $\mathrm{R}$ & R & $\begin{array}{l}\mathrm{R} \\
\mathrm{S}\end{array}$ & R & $\mathrm{R}$ & $\mathrm{R}$ & & & & & - & \\
\hline $\begin{array}{l}F \\
F \\
F \\
S \\
S \\
S \\
S \\
S\end{array}$ & $\begin{array}{l}\mathrm{R} \\
\mathrm{S} \\
\mathrm{R}\end{array}$ & $\begin{array}{l}S \\
S \\
R\end{array}$ & & $\begin{array}{c}\mathrm{L} \\
\mathrm{L} \\
\mathrm{L} \\
\mathrm{M} \\
\mathrm{M} \\
\mathrm{C} \\
\mathrm{F} \\
\mathrm{M}\end{array}$ & $\begin{array}{l}\mathrm{V} \\
\mathrm{C} \\
\mathrm{C} \\
\mathrm{C} \\
\mathrm{L} \\
\mathrm{M} \\
\mathrm{R}\end{array}$ & $\begin{array}{l}\mathrm{C} \\
\mathrm{M} \\
\mathrm{M} \\
\mathrm{S} \\
\mathrm{S}\end{array}$ & $\begin{array}{l}S \\
R \\
R \\
R\end{array}$ & $\mathbf{R}$ & $\begin{array}{l}\text { V } \\
\text { W } \\
X \\
\text { W } \\
\text { V } \\
\text { W } \\
\text { W } \\
\text { L } \\
\text { W }\end{array}$ & $\begin{array}{l}\mathrm{R} \\
\mathrm{A} \\
\mathrm{F} \\
\mathrm{F} \\
\mathrm{R}\end{array}$ & $\begin{array}{l}\text { S } \\
\\
\text { S } \\
\text { S } \\
\mathrm{F}\end{array}$ & $\begin{array}{l}\mathrm{R} \\
\mathrm{R} \\
\mathrm{S} \\
\mathrm{R}\end{array}$ & $\begin{array}{l}\mathrm{S} \\
\mathrm{R} \\
\mathrm{R}\end{array}$ & $\mathrm{R}$ & & $\begin{array}{l}F \\
R \\
S \\
R \\
R \\
\text { F } \\
\text { S } \\
\text { S } \\
F\end{array}$ & $\begin{array}{l}\mathrm{R} \\
\mathrm{R}\end{array}$ & $\begin{array}{l}\mathrm{R} \\
\mathrm{R} \\
\mathrm{R} \\
\mathrm{R} \\
\mathrm{R} \\
\\
\mathrm{R}\end{array}$ & & & $R$ & $\begin{array}{l}\mathrm{S} \\
\mathrm{R}\end{array}$ & & $\begin{array}{l}\mathrm{S} \\
\mathrm{S} \\
\mathrm{R}\end{array}$ & $\begin{array}{l}R \\
R\end{array}$ & & $\mathrm{R}$ & $\mathrm{R}$ & $\mathrm{R}$ & R & $\mathbf{R}$ & R & \\
\hline $\begin{array}{l}\mathrm{F} \\
\mathrm{M} \\
\mathrm{M} \\
\mathrm{F} \\
\mathrm{R}\end{array}$ & $\begin{array}{l}F \\
F \\
S\end{array}$ & $\begin{array}{c}\mathrm{R} \\
\mathrm{M} \\
\mathrm{F} \\
\mathrm{S}\end{array}$ & $\mathbf{R}$ & $\begin{array}{l}\mathrm{M} \\
\mathrm{C} \\
\mathrm{A} \\
\mathrm{C} \\
\mathrm{M}\end{array}$ & $\begin{array}{l}\text { C } \\
\text { A } \\
\text { L } \\
\text { C }\end{array}$ & $\begin{array}{l}\mathrm{R} \\
\mathrm{M} \\
\mathrm{R} \\
\mathrm{F}\end{array}$ & $\begin{array}{l}\mathrm{R} \\
\mathrm{F} \\
\mathrm{S} \\
\mathrm{F}\end{array}$ & $\begin{array}{l}\mathrm{R} \\
\mathrm{F} \\
\mathrm{R} \\
\mathrm{R}\end{array}$ & $\begin{array}{l}\text { W } \\
X \\
W \\
W \\
W \\
\text { V }\end{array}$ & $\begin{array}{l}\text { F } \\
\text { F } \\
\text { L } \\
\text { A } \\
\text { C }\end{array}$ & $\begin{array}{l}\mathrm{R} \\
\mathrm{R} \\
\mathrm{R} \\
\mathrm{S}\end{array}$ & $\begin{array}{l}\mathrm{S} \\
\mathrm{S} \\
\mathrm{R} \\
\mathrm{R}\end{array}$ & $\begin{array}{l}R \\
S\end{array}$ & & & $\begin{array}{l}\mathrm{F} \\
\mathrm{F} \\
\mathrm{S} \\
\mathrm{S}\end{array}$ & $\begin{array}{l}R \\
S \\
S\end{array}$ & & & & & & & $\begin{array}{l}\mathrm{S} \\
\mathrm{R} \\
\mathrm{S} \\
\mathrm{F} \\
\mathrm{R}\end{array}$ & $R$ & & $R$ & $\mathbf{R}$ & $R$ & R & R & $\mathbf{R}$ & $\mathbf{R}$ \\
\hline C & C & $\mathrm{F}$ & $\mathbf{R}$ & $\mathrm{L}$ & A & $\mathrm{A}$ & $F$ & $\mathrm{R}$ & V & A & $\mathrm{S}$ & F & $\mathbf{R}$ & $\mathrm{s}$ & & F & L & & $\mathrm{s}$ & $\mathrm{F}$ & w & $\mathrm{R}$ & & C & & & $\mathrm{S}$ & $\mathrm{M}$ & & $\mathrm{S}$ & $\mathrm{R}$ & & \\
\hline $\begin{array}{l}\mathrm{F} \\
\mathrm{R} \\
\mathrm{F} \\
\mathrm{S}\end{array}$ & $\begin{array}{l}\mathrm{R} \\
\mathrm{F} \\
\mathrm{C}\end{array}$ & $\begin{array}{l}\mathrm{R} \\
\mathrm{S} \\
\mathrm{F}\end{array}$ & $\begin{array}{l}\mathrm{S} \\
\mathrm{R} \\
\mathrm{R}\end{array}$ & $\begin{array}{l}\mathrm{L} \\
\mathrm{C} \\
\mathrm{L}\end{array}$ & $\begin{array}{l}\mathrm{V} \\
\mathrm{M} \\
\mathrm{A}\end{array}$ & $\begin{array}{l}\mathrm{R} \\
\mathrm{M}\end{array}$ & $\stackrel{\mathrm{F}}{\mathrm{M}}$ & $\begin{array}{l}\mathrm{R} \\
\mathrm{R} \\
\mathrm{F}\end{array}$ & $\begin{array}{l}\text { W } \\
\text { V } \\
\text { C } \\
\text { W }\end{array}$ & $\begin{array}{l}\text { C } \\
\text { A } \\
\text { A } \\
\text { A }\end{array}$ & $\begin{array}{l}\text { R } \\
\text { S }\end{array}$ & $\begin{array}{l}\mathrm{S} \\
\mathrm{R}\end{array}$ & $\begin{array}{l}\mathrm{R} \\
\mathrm{R} \\
\mathrm{R}\end{array}$ & & & $\mathrm{F}$ & $\begin{array}{l}\mathrm{F} \\
\mathrm{F} \\
\mathrm{F}\end{array}$ & $\begin{array}{l}\mathrm{R} \\
\mathrm{R}\end{array}$ & & $\begin{array}{l}S \\
R\end{array}$ & & $\mathrm{R}$ & & $\begin{array}{l}F \\
S \\
L\end{array}$ & $\begin{array}{l}\mathrm{R} \\
\mathrm{F}\end{array}$ & & $\begin{array}{l}\mathrm{S} \\
\mathrm{R}\end{array}$ & $\begin{array}{l}\mathrm{S} \\
\mathrm{R} \\
\mathrm{L}\end{array}$ & & $\begin{array}{l}\mathrm{R} \\
\mathrm{R}\end{array}$ & $\mathrm{R}$ & & $\mathbf{R}$ \\
\hline
\end{tabular}

Table 7 is a distribution chart of calcareous nannofossils recovered from Hole 838A. Floras in samples at $3.58 \mathrm{mbsf}(135-838 \mathrm{~A}-1 \mathrm{H}-3$, $58-59 \mathrm{~cm})$ and $9.2 \mathrm{mbsf}(135-838 \mathrm{~A}-2 \mathrm{H}-4,98-99 \mathrm{~cm})$ contain $E$. huxleyi and $H$. inversa; these samples are assigned to Zone CN15. Sample 135-838A-2H-CC (13.2 mbsf) yielded a flora that included extra abundant $E$. ovata and $P$. lacunosa; this is the highest observed occurrence of each of these species. The same sample included the lowest observed occurrence of $H$. inversa, which was common to abundant. Material in this sample was deposited in the late part of Subzone CN14a. The next datum encountered is the highest observed occurrence of $S$. pulcherrima in Sample 135-838A-4H-5, 93-94 cm (29.6 mbsf). The highest observed $H$. sellii is at 32 mbsf (Sample $135-838 \mathrm{~A}-5 \mathrm{H}-1,35-36 \mathrm{~cm}$ ). The single, poorly preserved $C$. macintyrei in this sample appeared to be reworked. Both of these datums fall within Subzone CN14a. The lowest observed specimens of $G$. oceanica and G. caribbeanica occur in samples from Core 135-838A$7 \mathrm{H}$, immediately overlying a section of coarse-grained sediment barren of nannofossils. This sample does not contain $C$. macintyrei, which should be present in the basal part of Zone CN14. The material recovered from Core $135-838 \mathrm{~A}-8 \mathrm{H}$, below the barren section, contains $C$. macintyrei but not $G$. oceanica, $G$. caribbeanica, or $D$. brouweri. This indicates deposition in Subzone CN13a. This series of assemblages indicates that nannofossils from the lowest part of Zone $\mathrm{CN} 14$ and all of Subzone CN13b were not recovered in this hole. The highest observed occurrences of $D$. brouweri and $D$. pentaradiatus are in Sample 135-838A-11H-2, 102-103 cm (91.7 mbsf). The assemblage in this sample indicates deposition in Subzone CN12c. Sparse floras in samples from the remainder of Hole 838A have no species, indicating deposition earlier than Subzone CN12c.
Table 8 is a distribution chart of nannofossils recovered in samples from Hole $838 \mathrm{~B}$. Recovery was generally poor from Hole 838B. All of the samples examined are from short sections or core-catcher samples. Floras are, however, generally quite well preserved and range from sparse to fair in abundance. Sample 135-838B-9R-CC (220.6 mbsf) yielded a rather sparse, poorly preserved flora including grainy, poorly preserved $D$. surculus, which may indicate deposition as old as Subzone $\mathrm{CN} 12 \mathrm{~b}$. The first sample yielding a really definitive flora, however, is Sample 135-838B-11R-1, 20-21 cm (230.4 mbsf). This flora includes Discoaster tamalis, which indicate deposition as old as Subzone CN12a. Rare Sphenolithus spp., which are present in this sample but not in any lower samples, are thought to be reworked. The remaining samples examined in this hole to Sample 135-838B-13R-CC contain similar floras, which indicate deposition in this same subzone.

\section{Site 839}

Site 839 is in the central Lau Basin about $70 \mathrm{~km}$ west of the Eastern Lau Spreading Center and approximately $225 \mathrm{~km}$ east of the Lau Ridge remnant arc (Fig. 1). Sediments collected at this site consist of clayey nannofossil ooze, turbiditic glassy sands and silts, pumiceous volcaniclastic gravels, and rare pyroclastic air-fall ashes. The sedimentary sequence is divided into three lithologic subunits that have no relation to biostratigraphic subdivisions. The oldest sediment encountered at this site is late Pliocene in age. The ages reported for this site, especially the position of the Pliocene/Pleistocene boundary in the present study, represent the only major departure from the biostratigraphic determinations as reported in the Leg 135 Initial Reports volume (Parson, Hawkins, Allan, et al., 1992). 
Table 4 (continued).

\begin{tabular}{|c|c|c|c|c|c|}
\hline Age & Zone & $\begin{array}{l}\text { Core, section, } \\
\text { interval (cm) }\end{array}$ & 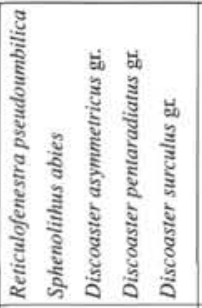 & 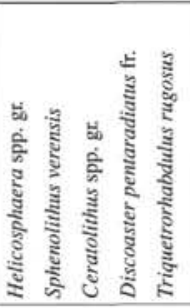 & 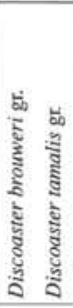 \\
\hline $\begin{array}{l}\text { Holocene- } \\
\text { Pleistocene }\end{array}$ & $\mathrm{CN} 15$ & $\begin{array}{l}135-835 \mathrm{~A}- \\
1 \mathrm{H}-1,35-37\end{array}$ & & & \\
\hline 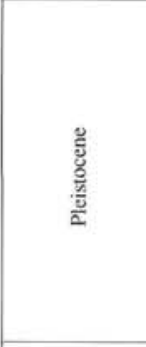 & $\mathrm{CN} 14$ & $\begin{array}{l}2 \mathrm{H}-4,37-38 \\
3 \mathrm{H}-7,18-19 \\
4 \mathrm{H}-5,52-53 \\
5 \mathrm{H}-7,10-11 \\
6 \mathrm{H}-3,30 \\
6 \mathrm{H}-4,60 \\
6 \mathrm{H}-5,30 \\
6 \mathrm{H}-6,60 \\
6 \mathrm{H}-6,90 \\
6 \mathrm{H}-6,120 \\
7 \mathrm{H}-1,30 \\
7 \mathrm{H}-2,40 \\
7 \mathrm{H}-3,70 \\
7 \mathrm{H}-4,90 \\
7 \mathrm{H}-5,60\end{array}$ & & & \\
\hline 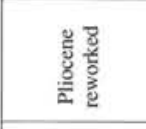 & \multirow{2}{*}{$\mathrm{CN} 12$} & $\begin{array}{l}7 \mathrm{H}-6,90 \\
7 \mathrm{H}-7,55 \\
8 \mathrm{H}-2,54 \\
8 \mathrm{H}-2,100 \\
8 \mathrm{H}-2,120\end{array}$ & & & \\
\hline 选 & & $\begin{array}{l}8 \mathrm{H}-2,144 \\
8 \mathrm{H}-2,145 \\
8 \mathrm{H}-3,58 \\
8 \mathrm{H}-5,120\end{array}$ & & & \\
\hline \multirow{3}{*}{$\begin{array}{l}\text { हूँ } \\
\frac{8}{2}\end{array}$} & $\mathrm{CN} 12$ & $\begin{array}{l}9 \mathrm{H}-5,70 \\
10 \mathrm{H}-5,41 \\
11 \mathrm{H}-5,88 \\
12 \mathrm{H}-3,72 \\
13 \mathrm{H}-4,125\end{array}$ & & & \\
\hline & $\mathrm{CN} I 1$ & $14 \mathrm{H}-1,100$ & L $\quad W \quad R \quad F \quad C$ & R $S$ & \\
\hline & $\mathrm{CN} 12$ & $\begin{array}{l}14 \mathrm{H}-7,31 \\
15 \mathrm{H}-5,140 \\
16 \mathrm{H}-4,129 \\
18 \mathrm{X}-1,1\end{array}$ & R $M \quad M$ & $\begin{array}{lll}S & S & \\
& & R\end{array}$ & $\mathrm{M} \mathrm{M}$ \\
\hline
\end{tabular}

Note: Key as in Table 2 .

Table 5. Distribution chart of calcareous nannofossils, Hole 836A.

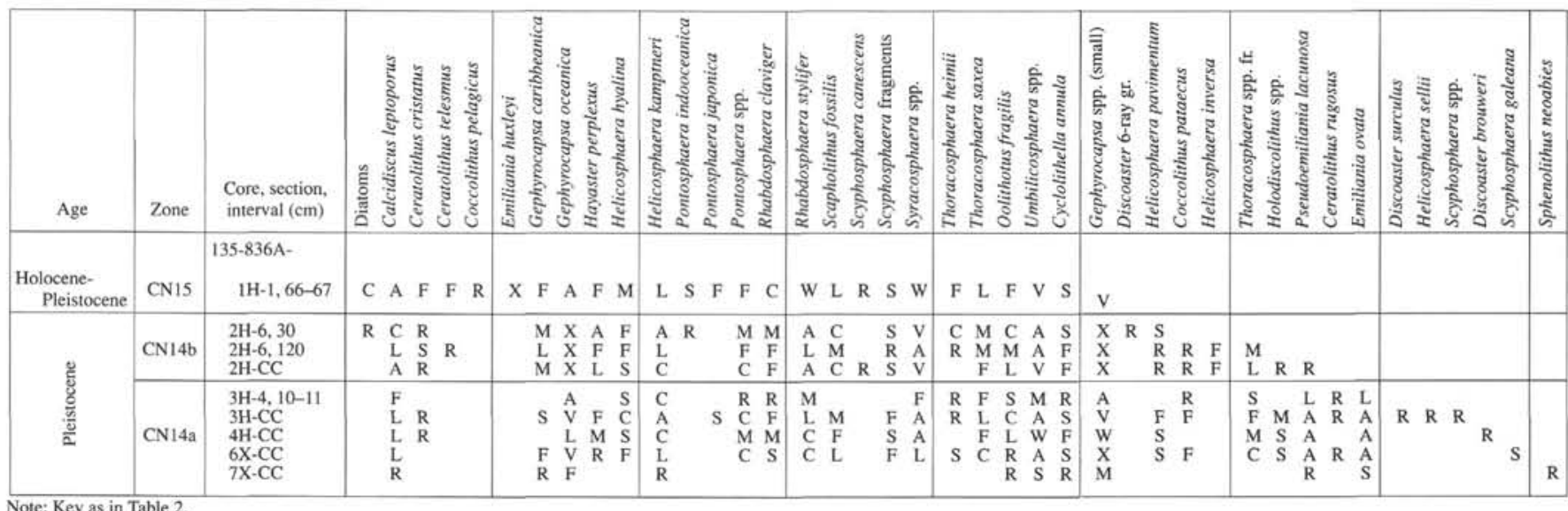

Table 9 is a distribution chart for Hole 839A. The shallowest sample examined from this site was one at $1.4 \mathrm{mbsf}$ (Sample 135$839 \mathrm{~A}-1 \mathrm{H}-1,139-140 \mathrm{~cm}$ ); this sample contained $E$. huxleyi and was assigned to Zone CN15. Sample 135-839A-1H-CC (4.5 mbsf) contained a rich flora that did not include $E$. huxleyi, P. lacunosa, or $E$. ovata. On this basis, the sample was assigned to Subzone CN14b. The sample did include the highest observed occurrence of $H$. inversa. The highest observed occurrence of $P$. lacunos $a$ and $E$. ovata plus the lowest observed occurrence of $H$. inversa was in Sample 135-839A-
$2 \mathrm{H}-4,120-121 \mathrm{~cm}(9.2 \mathrm{mbsf})$. This sample was assigned to Subzone $\mathrm{CN} 14 \mathrm{a}$. Two more datums from within that same subzone, the highest observed occurrences of $S$. pulcherrima and $H$. sellii, occur at 58.4 mbsf (Sample 135-839A-7H-5, $44-45 \mathrm{~cm}$ ) and $86.8 \mathrm{mbsf}$ (Sample 135-839A-10H-5, 31-32 cm), respectively. Some minor reworking of Pliocene taxa also occurred in this part of the sequence. The lowest observed $G$. oceanica at this site occurred in the core-catcher sample from Core 135-839A-12X (109.2 mbsf), which overlies a section of poor recovery extending to Core $135-839 \mathrm{~A}-15 \mathrm{X}$. The flora in this 
sample also did not include $C$. macintyrei, which would probably be present in sediments from near the initial occurrence of $G$. oceanica. The datum associated with this lowest occurrence, the top of Zone $\mathrm{CN} 13$, which approximates the Pliocene/Pleistocene boundary, could lie anywhere in this interval. Below Core $135-839 \mathrm{~A}-12 \mathrm{X}$ recovery was very poor; therefore, as in the lower part of the section at Site 838 , age assignments below this point must be regarded as spot determinations. Sample 135-839A-15X-CC (138.2 mbsf) yielded a flora including $C$. macintyrei and $G$. caribbeanica. This sample was assigned to Subzone CN13b. The flora in Sample 135-839A-20X-CC (186.2 mbsf) is the lowest sample containing $G$. caribbeanica. Floras recovered from below this point contain no further determinate nannofossils and were assigned to Subzone CN13b.

Table 10 is a chart of nannofossil taxa encountered in Sample 135-839B-17R-CC. This sample, which is from a thin lamina of sediment between basalt flows at $256.4 \mathrm{mbsf}$, contains a flora that includes rare $D$. brouweri. This sample is probably from Subzone CN12d.

\section{SUMMARY AND CONCLUSIONS}

Sediments recovered at the Lau Basin sites drilled during Leg 135 generally yielded well-preserved nannofossil floras. The main interest in biostratigraphic studies at these sites was to provide dates for the deposition of sediments immediately above basalts penetrated at five of the six sites. As expected, the age of these sediments increases away from the Central Lau Spreading Center and toward the Lau Ridge remnant arc. Age determinations for these basal sediments is unchanged from those presented in Parson, Hawkins, Allan, et al. (1992).

The sequence of nannofossil biostratigraphic events is as presented in Table 1. Of particular interest among these events are the extinction points of E.ovata, P. lacunosa, $H$. inversa, and $S$. pulcherrima. Extinction points, although generally considered less reliable datums than initial evolutionary occurrences, are of vital importance to paleontologists in the oil industry who work almost exclusively with drill cuttings rather than cores. The highest observed specimens $E$. ovata in Holes $834 \mathrm{~A}$ and $836 \mathrm{~A}$ occur above the highest observed specimens of $P$. lacunosa. This is similar to the pattern of extinctions for these taxa in the Gulf of Mexico, where I am usually employed. Although these species are generally lumped for sound biological reasons, perhaps a separation of the two is warranted for operational purposes. The extinction of $H$. inversa in Hole 838A falls above the base of Zone $\mathrm{CN} 15$; if this situation is widespread, it could possibly provide a useful datum for subdivision of that zone. In this study, the ranges of $H$. inversa and $E$. ovata sometimes overlap. This overlap was not reported by Shyu and Müller (1991). This may imply that the initial occurrence of $H$. inversa is within the very top part of Subzone CN14a but that the overlap of ranges may be small enough to require fortuitous sampling to pick it up. Gartner (1991) encouraged documentation of the extinction of $S$. pulcherrima. In three of the six sites, this datum falls within Subzone CN14a above the highest observed $H$. sellii.

\section{Systematic Paleontology}

\author{
Kingdom PLANTAE \\ Division CHRYSOPHYTA \\ Class COCCOLITHOPHYCEAE Rothmaler, 1951 \\ Order DISCOASTERACEAE Tan, 1927 \\ Family DISCOASTERACEAE Tan, 1927 \\ Genus DISCOASTER Tan, 1927 \\ Discoaster ono n. sp. \\ (Plate 1, Figs. 1-4)
}

Description. Asterolith minute $(1.5-4 \mu \mathrm{m})$ and symmetric with five bluntly pointed rays with the free length one half or less than the diameter of the central area. The central area is usually unornamented, but a slight knob is sometimes present. Between crossed nicols, the asterolith shows birefringence similar to that of Discoaster pentaradiatus.

Remarks. Discoaster ono is distinguished from $D$. pentaradiatus by its much smaller size and shorter bluntly pointed, nonbifurcated rays.
Holotype. The specimen illustrated in Plate 1, Figures 1-2, is designated as the holotype. This specimen, from Sample 135-834A-8H-2, $100 \mathrm{~cm}$, displays typical development of rays and central area.

Paratype. The specimen illustrated in Plate 1, Figures 3-4, is a designated paratype. This specimen, from Sample 135-838B-13R-CC, shows atypically well-developed rays and central area ornament.

Type level. late Pliocene, Zone CN12.

Type locality. Lau Basin Holes 834A and 838B. Similar specimens are present in Pliocene sediments in the Gulf of Mexico (M.J. Styzen, unpubl. data).

Epithet. For many years, this species has had the designation "Discoaster $6 "$ in the Shell Offshore Inc. nannofossil dictionary. It was suggested in the post-cruise meeting that the designation be retained but in a local language. The word "ono" is "six" in both Tongan and Fijian.

\section{Discoaster sp. A}

(Plate 1, Fig. 5)

Remarks. This medium-sized $(5-10 \mu \mathrm{m})$ discoaster possesses six nontapering rays of moderate length that terminate bluntly or with a slight notch. The central area is small and unornamented. The asterolith appears to be flat without the umbrella-shaped curvature seen in $D$. brouweri.

\section{ACKNOWLEDGMENTS}

I would like to thank Shell Offshore Inc. for supporting this research. Many individuals helped my participation in this project. Each knows what he or she contributed. They are Mary Berthonnaud, Anne Hill, Ralph Kerr, Brenda Melder, Ed Picou, and all the past and present members of the Shell Offshore Paleo staff. Thanks to all of you. I would also like to thank my reviewers Tony D'Agostino, Johanna Resig, and Steve Root for making this a better paper. Special thanks also to my mentors Lauralee Reugger and Peter Webb. Finally, thanks to my family, Renee, Andrea, and Valerie, for patience in my absence.

\section{REFERENCES*}

Berggren, W.A., Kent, D.V., and Van Couvering, J.A., 1985. The Neogene: Part 2. Neogene geochronology and chronostratigraphy. In Snelling, N.J. (Ed.), The Chronology of the Geological Record. Geol. Soc. London Mem., 10:211-260.

Bukry, D., 1973. Low-latitude coccolith biostratigraphic zonation. In Edgar, N.T., Saunders, J.B., et al., Init. Repts. DSDP, 15: Washington (U.S. Govt. Printing Office), 685-703.

1975. Coccolith and silicoflagellate stratigraphy, northwestern Pacific Ocean, Deep Sea Drilling Project Leg 32. In Larson, R.L., Moberly, R., et al., Init. Repts. DSDP, 32: Washington (U.S. Govt. Printing Office), 677-701.

Gartner, S., 1990. Neogene calcareous nannofossil biostratigraphy, Leg 116 (Central Indian Ocean). In Cochran, J.R., Stow, D.A.V., et al., Proc. ODP, Sci. Results, 116: College Station, TX (Ocean Drilling Program), 165-187.

, 1991. A note on Gephyrocapsa caribbeanica and amphora-shaped Scyphosphaera. INA Newsl., 13:103-104.

Martini, E., 1971. Standard Tertiary and Quaternary calcareous nannoplankton zonation. In Farinacci, A. (Ed.), Proc. 2nd Int. Conf. Planktonic Microfossils Roma: Rome (Ed. Tecnosci.), 2:739-785.

Okada, H., and Bukry, D., 1980. Supplementary modification and introduction of code numbers to the low-latitude coccolith biostratigraphic zonation (Bukry, 1973; 1975). Mar. Micropaleontol., 5:321-325.

Parson, L., Hawkins, J., Allan, J., et al., 1992. Proc. ODP, Init. Repts., 135: College Station, TX (Ocean Drilling Program).

Perch-Nielsen, K., 1985. Cenozoic calcareous nannofossils. In Bolli, H.M., Saunders, J.B., and Perch-Nielsen, K. (Eds.), Plankton Stratigraphy: Cambridge (Cambridge Univ. Press), 427-554.

Shyu, J.-P., and Müller, C.M., 1991. Calcareous nannofossil biostratigraphy of the Celebes and Sulu seas. In Silver, E.A., Rangin, C., von Breymann, M.T., et al., Proc. ODP, Sci. Results, 124: College Station, TX (Ocean Drilling Program), 133-158.

\footnotetext{
-Abbreviations for names of organizations and publication titles in ODP reference lists follow the style given in Chemical Abstracts Service Source Index (published by American Chemical Society).
}

Date of initial receipt: 25 June 1992

Date of acceptance: 8 December 1992

Ms 135SR-113 
Table 6. Distribution chart of calcareous nannofossils, Hole 837A.

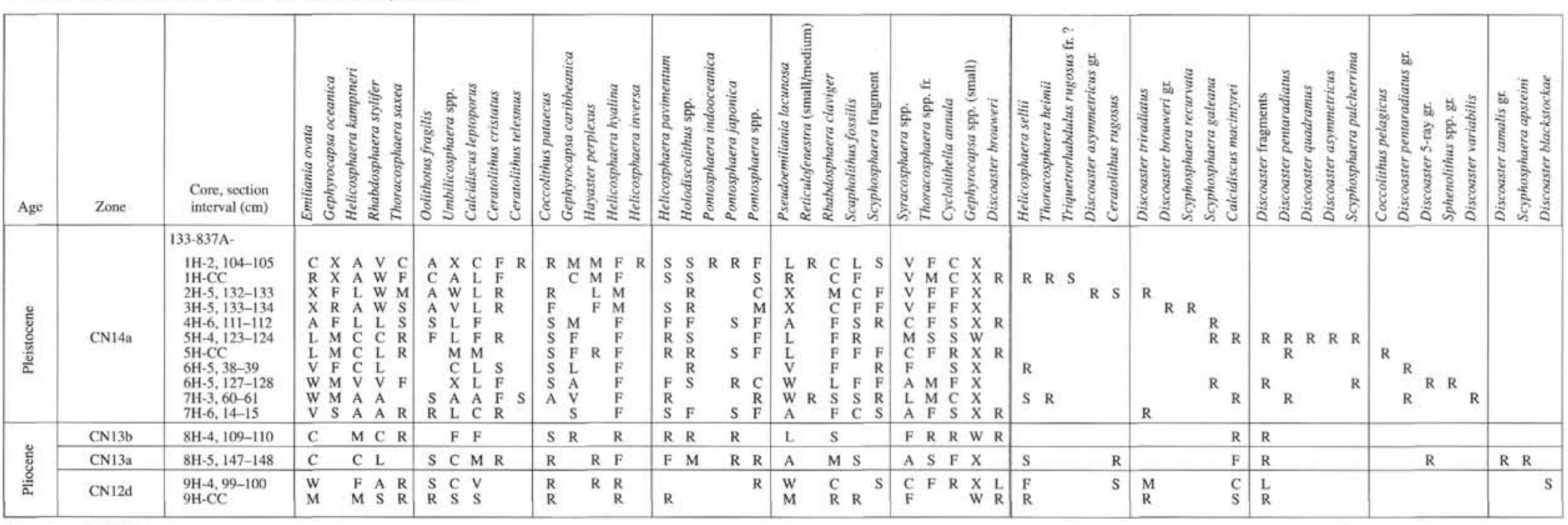

Table 7. Distribution chart of calcareous nannofossils, Hole $838 \mathrm{~A}$.

\begin{tabular}{|c|c|c|c|c|c|c|c|c|c|c|c|c|}
\hline Age & Zone & $\begin{array}{l}\text { Core, section, } \\
\text { interval (cm) }\end{array}$ & 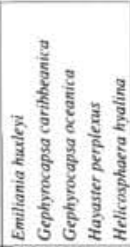 & 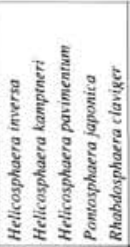 & 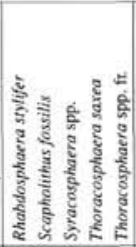 & 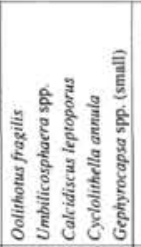 & 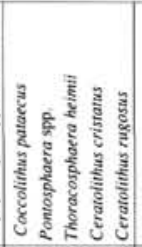 & 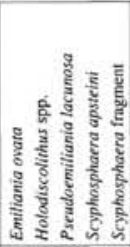 & 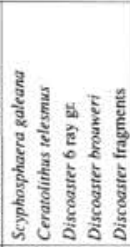 & 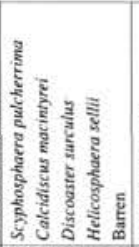 & 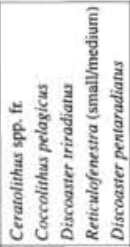 & 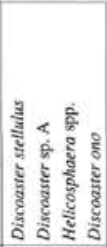 \\
\hline & & $135-838 \mathrm{~A}-$ & & & & & & & & & & \\
\hline 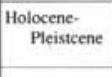 & CN IS & $\begin{array}{l}1 \mathrm{H}-3.58-59 \\
2 \mathrm{H}-4.98-99 \\
\end{array}$ & $\begin{array}{lllll}S & L & X & M & M \\
R & A & X & F & F\end{array}$ & $\begin{array}{lllll}F & C & S & R & M \\
S & L & F & M\end{array}$ & $\begin{array}{lllll}M & \text { M } & A & S & M \\
C & F & A & S & F\end{array}$ & $\begin{array}{lllll}C & L & R & F & X \\
L & W & M & M & X\end{array}$ & RS R & & & & & \\
\hline \multirow[t]{2}{*}{ 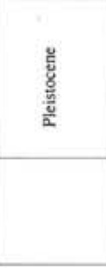 } & CN 14a & 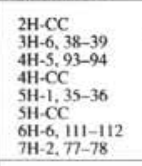 & 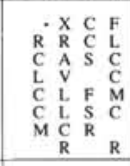 & 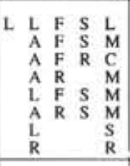 & $\begin{array}{lllll}\mathrm{V} & \mathrm{L} & \mathrm{W} & \mathrm{L} & \mathrm{L} \\
\mathrm{A} & \mathrm{C} & \mathrm{L} & \mathrm{S} & \mathrm{M} \\
\mathrm{A} & \mathrm{F} & \mathrm{A} & \mathrm{R} & \mathrm{M} \\
\mathrm{A} & \mathrm{F} & \mathrm{C} & \mathrm{R} \\
\mathrm{W} & \mathrm{F} & \mathrm{R} & \mathrm{R} \\
\mathrm{W} & \mathrm{F} & \mathrm{C} & \mathrm{R} \\
\mathrm{L} & \mathrm{L} & \mathrm{C} \\
\end{array}$ & $\begin{array}{lllll}A & X & A & S & X \\
\hat{L} & W & A & F & X \\
L & W & C & F & X \\
S & V & L & S & X \\
L & W & L & X \\
L & A & L & C & X \\
C & A & L & X \\
R & A & A & X \\
R & R & S\end{array}$ & 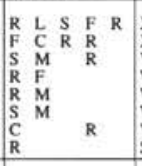 & 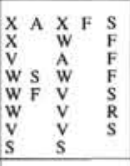 & $\begin{array}{c}F S R R \\
\\
\\
\\
R\end{array}$ & $\begin{array}{llll}R & & \\
& R & R & R \\
R & & R \\
& S\end{array}$ & & \\
\hline & $\mathrm{CN}$ 13a & 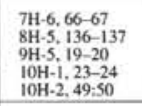 & MF & ${ }_{\mathrm{R}}^{\mathrm{A}} \mathrm{S} \quad \mathrm{M}$ & VF V R C & C V V $\underset{R}{L} F \underset{R}{F}$ & $S \underset{R}{F}$ & $\begin{array}{lll}w & \underset{R}{w} & s\end{array}$ & & $\begin{array}{r}\mathrm{F} \\
\mathrm{s} \\
\end{array}$ & s & \\
\hline 苋" & $\mathrm{CN} 12 \mathrm{c}$ & $\begin{array}{l}11 H-2,102-103 \\
1114-3,26-27 \\
14 X-1,31-32 \\
15 X-C C \\
16 X-1,55-56 \\
16 X-C C \\
17 X-C C\end{array}$ & & $\begin{array}{ll}\mathrm{L} & \mathrm{C} \\
\mathrm{R} \\
\mathrm{R} & \mathrm{R} \\
\mathrm{R} & \end{array}$ & $\begin{array}{lll}F & C & S \\
R & R & \\
R & R & \end{array}$ & $\begin{array}{lllll}F & C & L & F & w \\
R & & S & & S \\
& R & S & R \\
R & R & S & F \\
R & R & L \\
& R & R & F\end{array}$ & $\begin{array}{l}\mathrm{A} \\
\mathrm{R} \\
\mathrm{R} \\
\mathrm{S} \\
\mathrm{S} \\
\mathrm{S} \\
\mathrm{R}\end{array}$ & $\begin{array}{lll}\mathrm{V} & \mathrm{V} & \mathrm{R} \\
\mathrm{R} & \mathrm{C} & \\
\mathrm{S} & \mathrm{R} & \\
\mathrm{S} & \mathrm{F} & \\
\mathrm{S} & \mathrm{F} & \end{array}$ & $\begin{array}{lrr}R & A & L \\
& S & R \\
& R & R \\
& R & S \\
& & R\end{array}$ & $\begin{array}{ll}R & L \\
R & S \\
R & \end{array}$ & 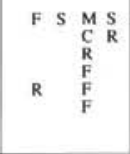 & $\begin{array}{lll}S & S_{R}^{S} & \\
& & \\
& & R\end{array}$ \\
\hline
\end{tabular}


Table 8. Distribution list of calcareous nannofossil, Hole 848B.

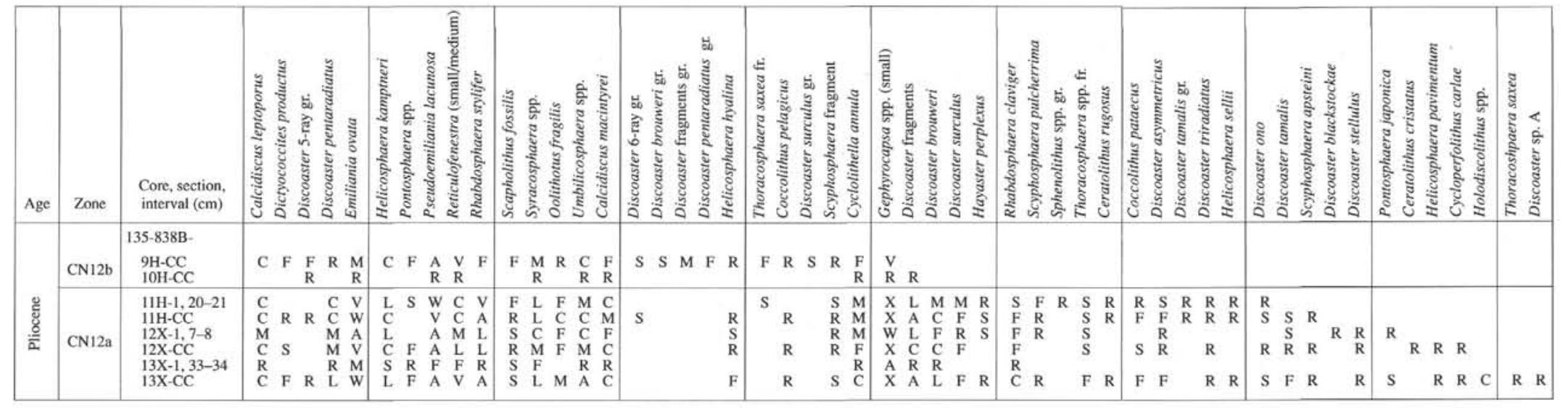

Note: Key as in Table 2.

Table 9. Distribution chart of calcareous nannofossils, Hole 839A.

\begin{tabular}{|c|c|c|c|c|c|c|c|c|c|c|c|c|}
\hline Age & Zone & $\begin{array}{l}\text { Core, Section, } \\
\text { interval (cm) }\end{array}$ & 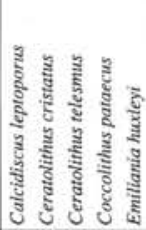 & 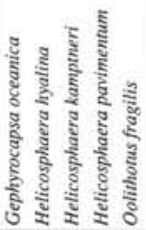 & 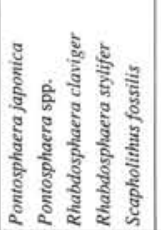 & 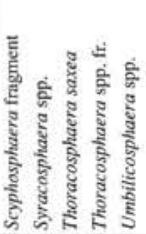 & 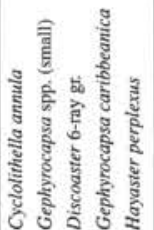 & 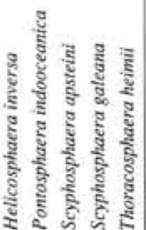 & 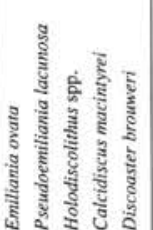 & 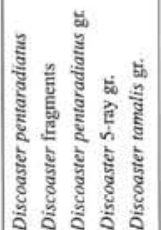 & 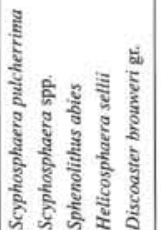 & 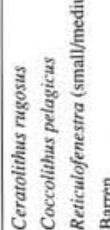 \\
\hline $\begin{array}{l}\text { olocene. } \\
\text { Pleistocene }\end{array}$ & CN15 & $\begin{array}{l}135-839 \mathrm{~A}- \\
1 \mathrm{H}-1,139-140\end{array}$ & A S S F X & WF VFF & R F L L W A & R W C R X & $\mathrm{MX}$ & & & & & \\
\hline & $\mathrm{CN} / 4 \mathrm{~b}$ & $\mathrm{HH}-\mathrm{CC}$ & CFS F & $X M \vee F A$ & 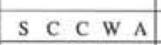 & $R \times M M X$ & $\mathrm{C} X \mathrm{RFC}$ & $R R R R$ & & & & \\
\hline $\begin{array}{l}\frac{0}{5} \\
\frac{8}{2} \\
\frac{3}{2}\end{array}$ & $\mathrm{CN} 14 \mathrm{a}$ & 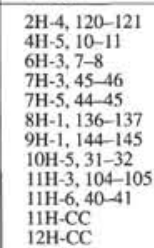 & 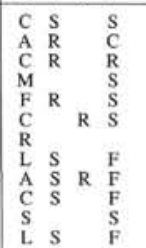 & 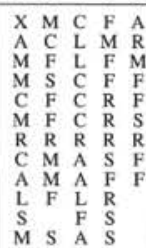 & 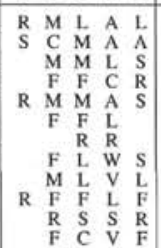 & 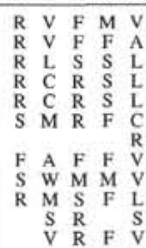 & $\begin{array}{llll}\mathrm{F} & \mathrm{X} & \mathrm{F} & \mathrm{F} \\
\mathrm{F} & \mathrm{W} & \mathrm{R} \\
\mathrm{R} & \mathrm{R} & \mathrm{R} \\
\mathrm{R} & \mathrm{C} & \mathrm{C} & \mathrm{F} \\
\mathrm{S} & \mathrm{X} & \mathrm{F} & \mathrm{P} \\
\mathrm{S} & \mathrm{X} & \mathrm{F} & \mathrm{R} \\
\mathrm{R} & \mathrm{X} & \mathrm{M} \\
\mathrm{S} & \mathrm{C} & \mathrm{R} \\
\mathrm{S} & \mathrm{X} & \mathrm{L} & \mathrm{R} \\
\mathrm{R} & \mathrm{X} & \mathrm{L} \\
\mathrm{F} & \mathrm{X} & \mathrm{C} \\
\mathrm{R} & \mathrm{A} & \mathrm{S} \\
\mathrm{R} & \mathrm{A} & \mathrm{S}\end{array}$ & 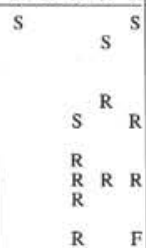 & 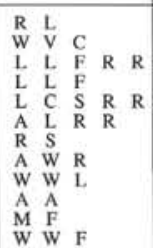 & 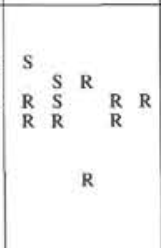 & 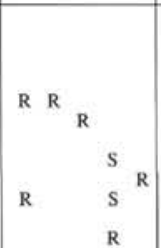 & ${ }^{R}$ \\
\hline \multirow{2}{*}{ 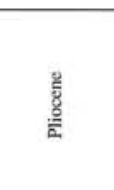 } & $\mathrm{CN} 13 \mathrm{~b}$ & 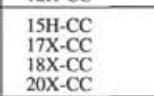 & 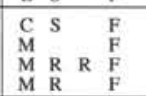 & 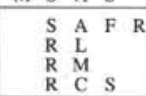 & $\begin{array}{|lllll|}R & F & R & A & M \\
& S & S & M & R \\
R & R & R & R \\
R & R & R & R\end{array}$ & 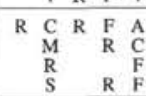 & $\begin{array}{lll}F & X & \\
S & \text { P } \\
\text { R } & \text { V } & S \\
\text { W } & S \\
\text { W } & \text { F }\end{array}$ & & 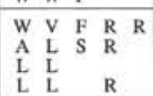 & & $\begin{array}{l}\mathrm{R} \\
\mathrm{R} \\
\mathrm{S}\end{array}$ & \\
\hline & $\mathrm{CN}_{3} 3 \mathrm{a}$ & 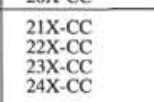 & $\begin{array}{|ll|}\mathrm{S} & \mathrm{R} \\
\mathrm{F} & \mathrm{R} \\
\mathrm{F} & \mathrm{F} \\
\end{array}$ & $\begin{array}{lll}R & \text { F } & \text { F } \\
\mathrm{S} & \mathrm{R} \\
\mathrm{S} & \mathrm{S}\end{array}$ & $\begin{array}{ll}S_{R}^{R} & R \\
R\end{array}$ & 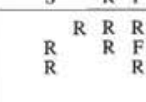 & $\begin{aligned} \underset{\mathrm{W}}{\mathrm{W}} \\
\mathrm{R}\end{aligned}$ & & $\begin{array}{lll} & & \text { S } \\
C & \text { L } & \text { S } \\
\text { V } & \text { L } & \text { M }\end{array}$ & & $\mathrm{R}$ & 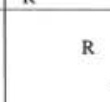 \\
\hline
\end{tabular}


Table 10. Distribution chart of calcareous nannofossils, Hole 839B.

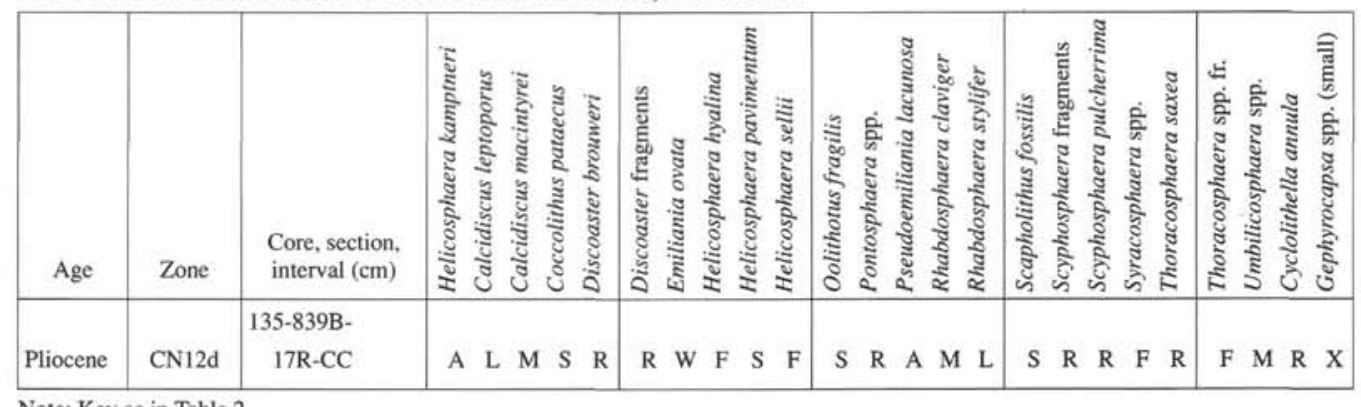

Note: Key as in Table 2. 


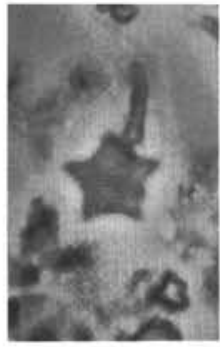

1

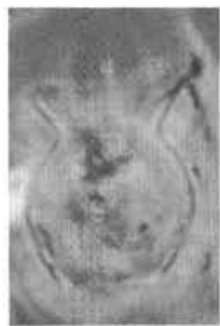

6

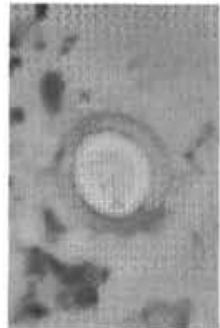

10

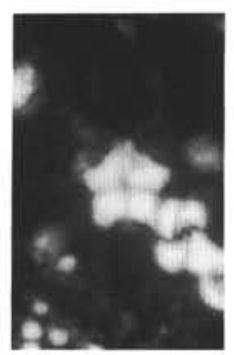

2

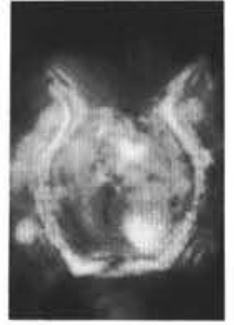

7

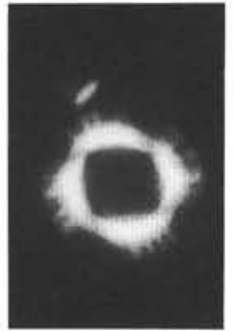

11

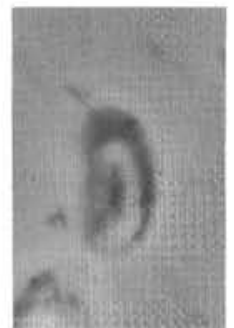

$15 a$

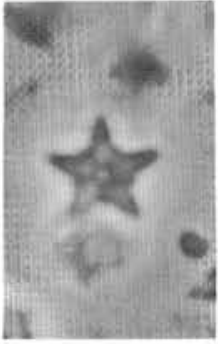

3

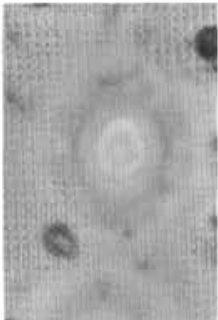

8

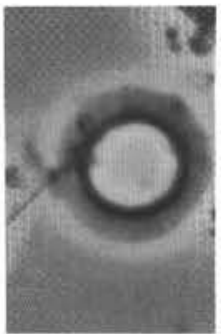

12

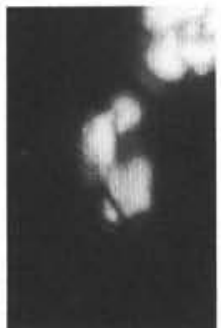

$15 b$

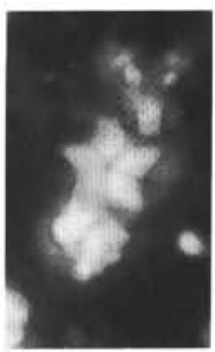

4

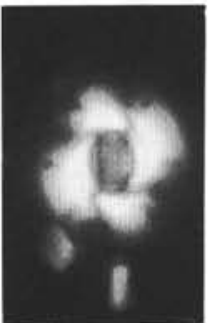

9

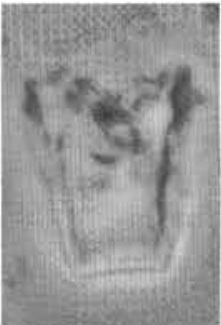

13

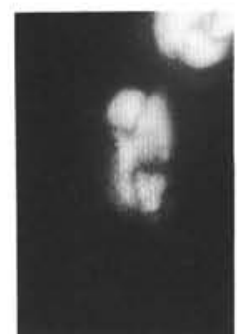

$15 c$

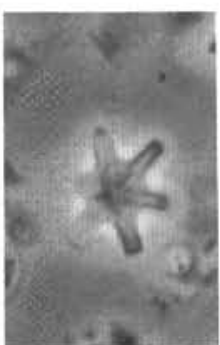

5

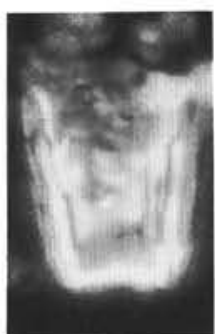

14

Plate 1. All specimens are magnified 2800x. 1-2. Discoaster ono n. sp. holotype, Sample 135-834A-8H-3, $40 \mathrm{~cm}$. 3, 4. Discoaster ono n. sp. paratype, Sample 135-838B-13R-CC. 5. Discoaster sp. A, Sample 135-838B-13R-CC. 6, 7. Scyphosphaera pulcherrima Deflandre, Sample 135-838B-13R-CC. 8,9. Emiliania ovata Bukry, Sample $135-836 \mathrm{~A}-3 \mathrm{H}-4,60 \mathrm{~cm}$. 10-11. Pseudoemiliania lacunosa (Kamptner) Gartner, Sample 135-836A-3H-4, $60 \mathrm{~cm}$. 12. Cyclolithella annula (Cohen) Boudreaux and Hay, Sample 135-835A-6H-4, 60 cm. 13-14. Scyphosphaera galeana Kamptner, Sample 135-836A-3H-CC. 15. Helicosphaera pavamentum Okada and McIntyre; (15a) phase contrast; (15b) view parallel to crossed nicols; (15c) view $45^{\circ}$ to crossed nicols; Sample $135-838 \mathrm{~A}-1 \mathrm{H}-3,58-59 \mathrm{~cm}$. 


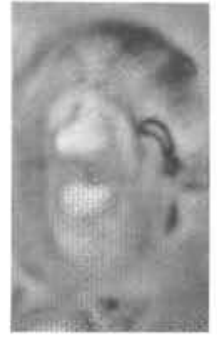

1

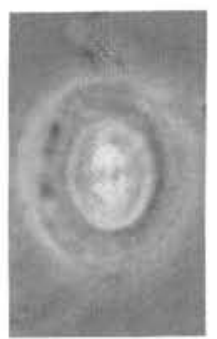

5

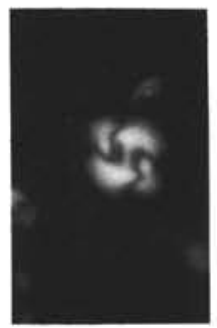

9

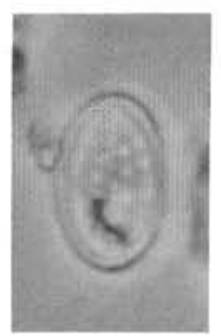

$13 a$

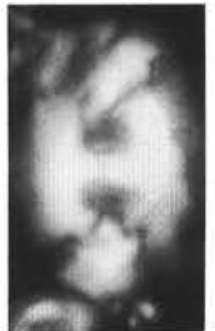

2

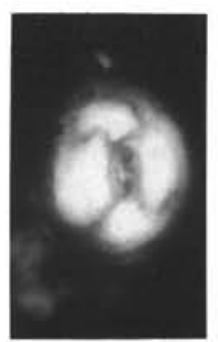

6

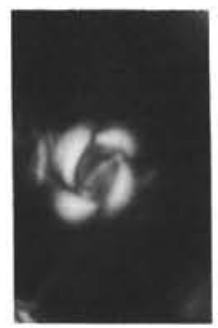

10

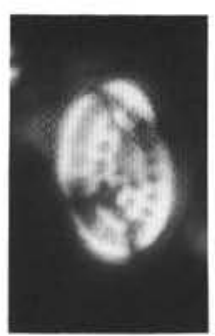

13b

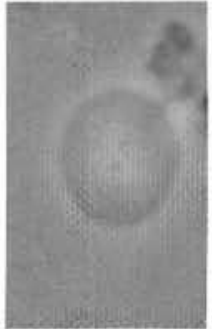

3

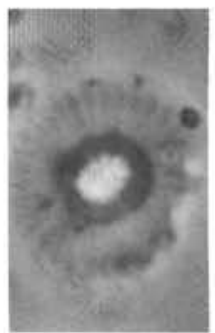

7

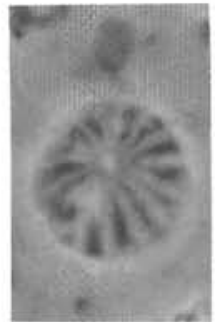

11

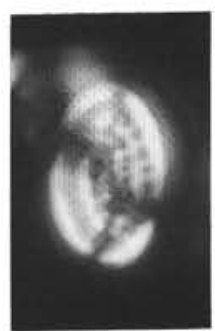

$13 c$

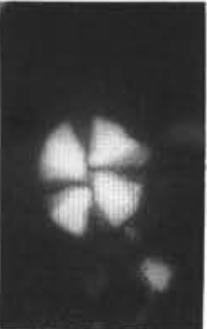

4

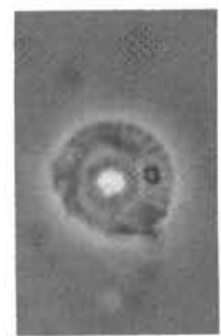

8

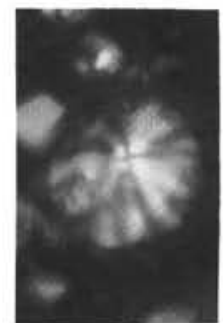

12

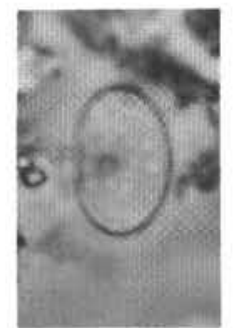

$14 a$

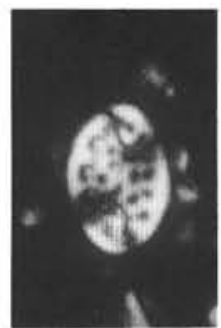

14b

Plate 2. All specimens are magnified 2800×. 1-2. Helicosphaera inversa Gartner, Sample 135-836A-2H-6, $30 \mathrm{~cm}$. 3. Coccolithus pataecus Gartner, Sample 135-838A-11H-3, 26-27 cm. 4. Coccolithus pataecus Gartner, Sample 135-837A-7H-3, 60 cm. 5-6. Coccolithus pelagicus (Wallich) Schiller, Sample 135-838B-13R-CC. 7. Cycloperfolithus carlae Lehotayova and Priewalder, Sample 135$834 \mathrm{~A}-8 \mathrm{H}-3,40 \mathrm{~cm}$. 8. Cycloperfolithus carlae Lehotayova and Priewalder, Sample $135-834 \mathrm{~A}-9 \mathrm{H}-1,10 \mathrm{~cm}$. 9. Gephyrocapsa oceanica Kamptner, Sample 135-836A-2H-6, $30 \mathrm{~cm}$. 10. Gephyrocapsa caribbeanica Boudreaux and Hay, Sample 135-836A-2H-6, $30 \mathrm{~cm}$. 11-12. Oolithotus fragilis (Lohman) Martini and Müller, Sample 135-836A-3H-CC. 13. Pontosphaera indooceanica epek; (13a) phase contrast; (13b) view parallel to crossed nicols; (13c) view $45^{\circ}$ to crossed nicols; Sample $135-836 \mathrm{~A}-2 \mathrm{H}-6,30 \mathrm{~cm}$. 14. Pontosphaera indooceanica Cepek; (14a) phase contrast; (14b) view parallel to crossed nicols; Sample 135-836A-2H-6, $30 \mathrm{~cm}$. 


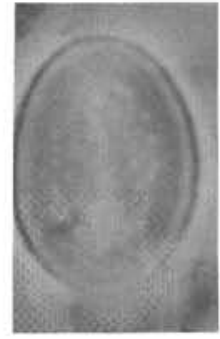

1

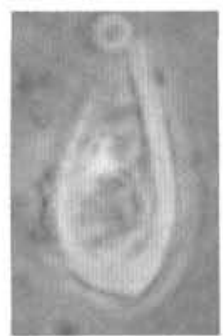

5

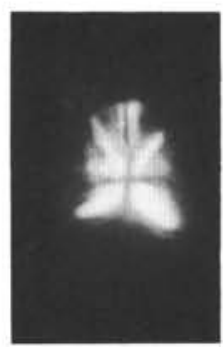

$8 \mathbf{a}$

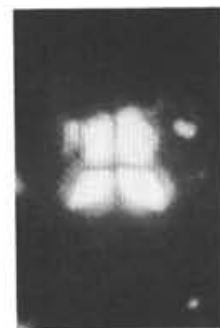

$10 \mathrm{a}$

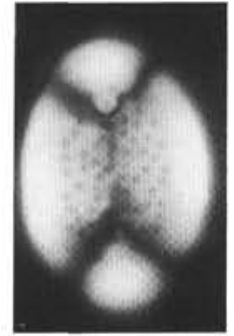

2

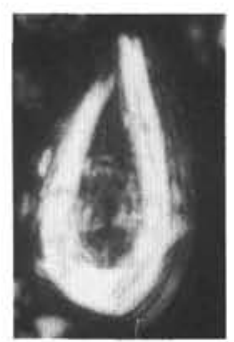

6

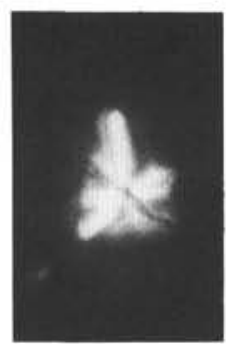

$8 \mathrm{~b}$

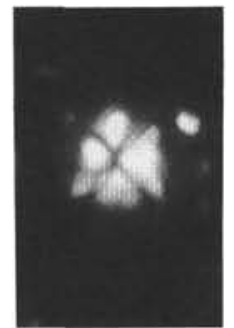

$10 \mathrm{~b}$

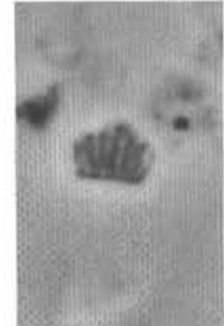

3

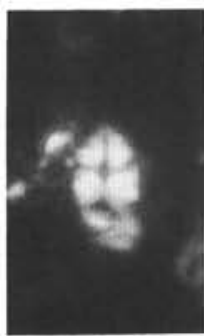

$7 a$

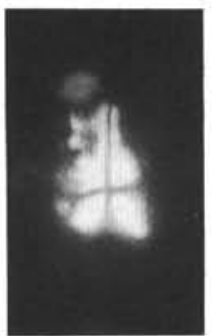

$9 a$

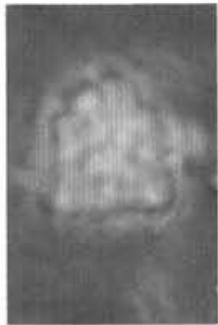

$11 \mathrm{a}$

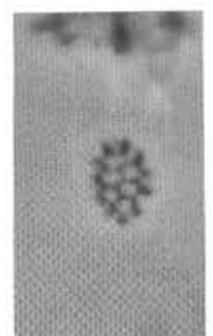

4

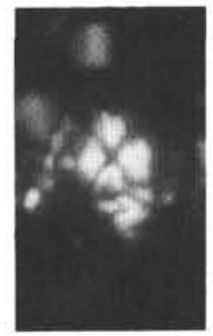

$7 b$

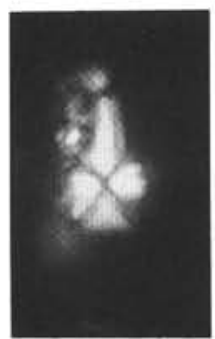

9b

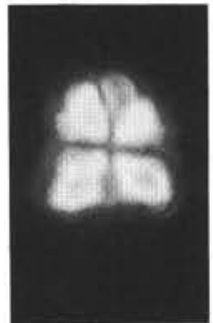

$11 \mathrm{~b}$

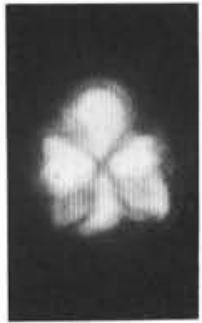

$11 c$

Plate 3. All specimens are magnified 2800x, 1-2. Pontosphaera japonica (Takayama) Nishida, Sample 135-835A-1H-1, 35-37 cm. 3-4. Holodiscolithus sp., Sample 135-837A-5H-4, 123-124 cm. 5-6. Ceratolithus telesmus Norris, Sample 135-837A-7H-3, 60 cm. 7. Sphenolithus neoabies Bukry and Bramlette; (7a) parallel to crossed nicols; (7b) $45^{\circ}$ to crossed nicols; Sample $135-834 \mathrm{~A}-9 \mathrm{H}-1$, $10 \mathrm{~cm}$. 8. Sphenolithus abies Deflandre; (8a) parallel to crossed nicols; (8b) $45^{\circ}$ to crossed nicols; Sample $135-834 \mathrm{~A}-9 \mathrm{H}-1$, $10 \mathrm{~cm}$. 9. Sphenolithus abies Deflandre; (9a) parallel to crossed nicols; (9b) view $45^{\circ}$ to crossed nicols; Sample 135-834A-9H-1, $10 \mathrm{~cm} . \quad 10$. Sphenolithus compactus Backman; (10a) parallel to crossed nicols; (10b) view $45^{\circ}$ to crossed nicols; Sample $135-834 \mathrm{~A}-16 \mathrm{X}-1$, $111-112 \mathrm{~cm}$. 11. Sphenolithus moriformus (Brönnimann and Stradner) Bramlette and Wilcoxon; (11a) phase contrast; (11b) parallel to crossed nicols; (11c) view $45^{\circ}$ to crossed nicols; Sample $135-834 \mathrm{~A}-16 \mathrm{X}-1,111-112 \mathrm{~cm}$. 